\title{
EXTREMAL LENGTH AND HARMONIC FUNCTIONS ON RIEMANN SURFACES
}

\author{
BY
}

\author{
CARL DAVID MINDA( $\left.{ }^{1}\right)$
}

\begin{abstract}
Expressions for several conformally invariant pseudometrics on a Riemann surface $R$ are given in terms of three new forms of reduced extremal distance. The pseudometrics are defined by means of various subclasses of the set of all harmonic functions on $R$ having finite Dirichlet integral. The reduced extremal distance between two points is defined on $R$, on the Alexandroff onepoint compactification of $R$ and on the Kerékjártó-Stö̈low compactification of $R$. These reduced extremal distances are computed in terms of harmonic functions having specified singularities and boundary behavior. The key to establishing this connection with harmonic functions is a general theorem dealing with extremal length on a compact bordered Riemann surface and its extensions to noncompact bordered surfaces. These results are used to obtain new tests for degeneracy in the classification theory of Riemann surfaces. Finally, some of the results are illustrated for a hyperbolic simply connected Riemann surface.
\end{abstract}

Introduction. Three new forms of reduced extremal distance are defined for a Riemann surface $R$. These are related to the concept of the same name defined by Ahlfors and Beurling [2] and to their notion of perimeter [1]. For example, the reduced extremal distance $\lambda\left(\zeta_{1}, \zeta_{2}\right)$ between two points $\zeta_{1}, \zeta_{2}$ on $R$ is defined by removing disks of radii $r_{1}, r_{2}$ about $\zeta_{1}, \zeta_{2}$, respectively, considering the extremal length of the family of all curves on $R$ joining the boundaries of these disks and then adding the factor $(1 / 2 \pi) \log \left(r_{1} r_{2}\right)$ in order to obtain a quantity with a finite limit $\lambda\left(\zeta_{1}, \zeta_{2}\right)$ as $r_{1}, r_{2} \rightarrow 0 . \lambda^{\prime}\left(\zeta_{1}, \zeta_{2}\right)$ and $\lambda^{*}\left(\zeta_{1}, \zeta_{2}\right)$ are defined in a similar manner except that the curves between the disks are taken on the Alexandroff onepoint compactification $R^{\prime}$ and the Kerékjártó-Stoïlow compactification $R^{*}$, respectively. These reduced extremal distances are computed in terms of harmonic functions having prescribed singularities and boundary behavior (Theorems 5, 6). The key to establishing this connection with harmonic functions is a general result (Theorem 1) dealing with extremal distance on compact bordered Riemann surfaces

Received by the editors August 4, 1971.

AMS 1970 subject classifications. Primary 31A05, 31A15; Secondary 30A48.

Key words and phrases. Extremal length, reduced extremal distance, harmonic function, conformally invariant pseudometric, Alexandroff one-point compactification, KerékjártóStoillow compactification, normal operator, principal function, classification theory.

(1) The majority of results in this paper were part of the author's doctoral dissertation which was written at the University of California, San Diego, under the direction of Professor Burton Rodin. This research was partially supported by U. S. Air Force Grant AFOSR. 68-1514. The manuscript was prepared with partial assistance from National Science Foundation Grant GP-27275. 
and its extensions (Theorems 2, 3, 4) to noncompact bordered surfaces which generalize a theorem of Marden and Rodin [4].

Next, several conformally invariant pseudometrics are defined from classes of harmonic functions. Let $H D(R)$ be the class of all harmonic functions $u$ on $R$ having finite Dirichlet integral $D(u)$. A conformally invariant pseudometric $d_{H D}\left(\zeta_{1}, \zeta_{2}\right)$ is introduced on $R$ by taking the supremum of $\left|u\left(\zeta_{1}\right)-u\left(\zeta_{2}\right)\right|$ over all $u \in H D(R)$ with $D(u) \leq 1$. Two other pseudometrics, $d_{K D}$ and $d_{H M}$, are defined analogously by employing the subclasses $K D(R)$ and $H M(R)$ of $H D(R) . K D(R)$ is the subset of $u \in H D(R)$ such that the integral of $* d u$ over every dividing cycle $\gamma$ on $R$ is 0 . A cycle $\gamma$ on $R$ is called dividing if for every compact set $E \subset R$ there is a cycle in $R \sim E$ homologous to $\gamma$.HM(R), the space of harmonic measures, is the orthogonal complement of $K D(R)$ in the Hilbert space $H D(R)$.

Then (Theorem 7) it is shown that $d_{H D}=\lambda-\lambda^{\prime}, d_{K D}=\lambda-\lambda^{*}$ and $d_{H M}=$ $\lambda^{*}-\lambda^{\prime}$, which provides a geometric extremal length interpretation of the various pseudometrics. Finally, this result is applied to derive tests for degeneracy in the classification theory of Riemann surfaces. A Riemann surface $R$ is of class $O_{H D}, O_{K D}, O_{H M}$ if the class $H D(R), K D(R), H M(R)$, respectively, contains only constant functions. It is proved (Theorems 8,9$)$ that $R \in O_{H D}\left(O_{K D}, O_{H M}\right)$ if and only if the extremal distance between any two compact subsets of $R$ is the same whether computed on $R$ or $R^{\prime}\left(R\right.$ or $R^{*}, R^{\prime}$ or $\left.R^{*}\right)$. The result for $O_{K D}$ is due to Rodin [6].

Finally, several explicit computations of the reduced extremal distances and a conformally invariant metric are given for hyperbolic simply connected Riemann surfaces.

1. Preliminaries. For general results about Riemann surfaces see AhlforsSario [3].

(1.1) At times we shall require the existence and perhaps uniqueness of a harmonic function having certain singularities and prescribed behavior in a neighborhood of the ideal boundary $\beta$ of a Riemann surface $R$. This will usually follow from the main existence theorem of the theory of normal operators ([3, Theorem $3 A$, p. 154], [8, Theorem 2B, p. 41]). A knowledge of the particular normal operators $L_{0},(I) L_{1}$ and $(Q) L_{1}$ is necessary [8, pp. 46-51]. These operators give precise meaning to the boundary behavior of a harmonic function on $\beta$. For example, if $u$ is harmonic on $R$, then $u=L_{0} u$ in a neighborhood of $\beta$ is akin to having a vanishing normal derivative on the boundary; that is, $* d u=0$ along the boundary where $* d u=-u_{y} d x+u_{x} d y$ is the star conjugate of the differential form $d u=u_{x} d x$ $+u_{y} d y$ on $R$. Likewise, $u=(I) L_{1} u$ near $\beta$ means that $u$ is constant on $\beta$ and $\int_{\beta} * d u=0$, while $u=(Q) L_{1} u$ implies that $u$ is constant on each contour of $\beta$ and the integral of $* d u$ over each contour of $\beta$ vanishes. Of course, both of these statements only hold in an appropriate limiting sense on a general Riemann surface, 
but they are to be taken literally on a compact bordered Riemann surface. Recall that the Dirichlet integral of a harmonic function $u$ on a Riemann surface $R$ is

$$
D_{R}(u)=D(u)=\iint_{R}\left(u_{x}^{2}+u_{y}^{2}\right) d x d y .
$$

The subscript $R$ is frequently omitted when one fixed Riemann surface is under consideration.

(1.2) The notion of extremal length for a family of chains on a Riemann surface will be employed. For a treatment of this topic see Rodin-Sario [8, pp. 121-132] and Sario-Oikawa [9, Appendix I]. A curve on $R$ is a continuous mapping $c: I \rightarrow$ $R$ where $I$ is an open, closed or half-open interval on the real line. The curve $c$ is locally rectifiable if the restriction of $c$ to any compact subinterval of $I$ is rectifiable. A locally rectifiable chain on $R$ is a countable formal sum $c=\Sigma n_{j} c_{j}$ where $n_{j}$ is an integer and $c_{j}$ is a locally rectifiable curve. Henceforth, all curves and chains are assumed to be locally rectifiable. Let $d s=\rho(z)|d z|$ be a linear density on $R$ and let $c$ be a locally rectifiable curve. $\int_{c} d s$ is defined to be the supremum of the integrals of $d s$ over all compact subpaths of $c$. For a locally rectifiable chain $c=\Sigma n_{j} c_{j}$ set

$$
\int_{c} d s=\sum\left|n_{j}\right| \int_{c_{j}} d s
$$

This integral is called the $d s$ or $\rho$ length of $c$ and is denoted by $L(c, d s)=$ $L(c, \rho)$. The $d s$ area of $R$ is

$$
A(R, d s)=A(d s)=\iint_{R} \rho^{2}(z) d x d y .
$$

Given a family $\mathcal{F}$ of locally rectifiable chains on $R$ and a linear density $d s$, set

$$
L(\mathcal{F}, d s)=\inf _{c \in \mathcal{F}} L(c, d s) .
$$

With the convention that $0 / 0=\infty / \infty=0$, the quantity $\lambda(\mathcal{F})=\sup \left(L^{2}(\mathcal{F}, d s) / A(R, d s)\right)$ is called the extremal length of $\mathfrak{F}$; the supremum is taken over all linear densities $d s$ on $R$.

2. Extremal distance on compact bordered surfaces. Let $\bar{R}$ be a compact bordered Riemann surface with interior $R$ and border $\partial \bar{R}$. Suppose that the contours of $\partial \bar{R}$ are partitioned into disjoint sets $\alpha_{0}, \alpha_{1}, \gamma_{0}, \gamma_{1}$ with $\alpha_{0}, \alpha_{1}$ nonempty. When $\gamma_{1}$ is nonvoid let the contours in $\gamma_{1}$ be divided into nonempty disjoint sets, say $\beta_{1}, \beta_{2}, \cdots, \beta_{m}$. Each $\beta_{j}$ is called a part of $\gamma_{1}$. Thus, $\left\{\alpha_{0}, \alpha_{1}, \gamma_{0}, \beta_{1}\right.$, $\left.\cdots, \beta_{m}\right\}$ represents a partition of $\partial \bar{R}$. Let $\mathcal{F}$ be the class of finite chains in $\bar{R} \sim$ $\gamma_{0}$ which join $\alpha_{0}$ to $\alpha_{1}$, possibly via some of the parts of $\gamma_{1}$. More precisely, a finite chain $\sum_{j=1}^{k} c_{j}$ belongs to $\mathfrak{F}$ if and only if either $k=1$ and $c_{1}$ is a curve in $\bar{R} \sim \gamma_{0}$ which connects $\alpha_{0}$ and $\alpha_{1}$ or $k \geq 2$ and the $c_{j}:[0,1] \rightarrow \bar{R} \sim \gamma_{0}$ are 
curves with $c_{1}(0) \in \alpha_{0}, c_{k}(1) \in \alpha_{1}$, and for $1 \leq j \leq k-1$ the points $c_{j}(1)$ and $c_{j+1}(0)$ belong to the same part of $\gamma_{1}$. If the points in each part of $\gamma_{1}$ were identified and $\bar{R}$ given the induced quotient topology, then each chain in $\mathcal{F}$ could be viewed as a continuous curve in this quotient space from $\alpha_{0}$ to $\alpha_{1}$. In case $\gamma_{1}=\emptyset$ the extremal length $\lambda(\mathcal{F})$ is customarily called the extremal distance between $\alpha_{0}$ and $\alpha_{1}$. The same designation for $\lambda(\mathfrak{F})$ will still be used when $\gamma_{1} \neq \emptyset$.

The following theorem generalizes a result of Marden and Rodin ([4, Lemma III. 1.1 , p. 250], [8, Theorem 1D, p. 1241); the proof is an adaptation of theirs. They partition $\gamma_{1}$ into its constituent contours; that is, each $\beta_{j}$ is a single contour of $\gamma_{1}$. The extension allows $\gamma_{1}$ to be separated into sets of contours in an arbitrary manner.

Theorem 1. $\lambda(\mathfrak{F})=D_{\bar{R}}(u)^{-1}$ where $u$ is the barmonic function on $\bar{R}$ such that $u=0$ on $\alpha_{0}, u=1$ on $\alpha_{1}, * d u=0$ along $\gamma_{0}, u$ is a constant $c_{j}, 0 \leq c_{j} \leq 1$, on each $\beta_{j}(1 \leq j \leq m)$ and $\int_{\beta_{j}} * d u=0$ for all $j$.

Proof. The existence and uniqueness of $u$ follows from the construction of the normal operators $L_{0}$ and $L_{1}$ [8, pp. 46-48]. An appropriate direct sum of the two operators $L_{0}$ and $L_{1}$ is required to produce the desired boundary behavior on $\gamma_{0}$ and $\gamma_{1}$.

First we show that $\lambda(\mathfrak{F}) \geq D(u)^{-1}$ by using the particular linear density $d s=$ $|d u+i * d u|=\left|u_{x}-i u_{y}\right||d z|$. The $d s$-area of $\bar{R}$ is $A(d s)=D(u)$. Observe that $d s$ $\geq|d u|$ and that the variation of $u$ along any chain in $\mathcal{F}$ is at least 1 , the difference between the values of $u$ on $\alpha_{0}$ and $\alpha_{1}$. Consequently, $L(\mathcal{F}, d s) \geq 1$ and

$$
D_{\bar{R}}(u)^{-1} \leq L^{2}(\mathcal{F}, d s) A(d s)^{-1} \leq \lambda(\mathcal{F}) .
$$

In order to establish the reverse inequality we shall consider chains which are locally the level curves of a harmonic conjugate $u^{*}$ of $u$; that is, curves along which $* d u=0$. We shall find a parametrization of these level curves such that all but finitely many belong to $\mathcal{F}$.

It is necessary to orient and parametrize the boundary curves of $\bar{R}$. Orient the contours of $\partial \bar{R}$ so that $\bar{R}$ lies to the left. All of the contours in $a_{j}(j=0,1)$ shall be parametrized by a single function $a_{j}(t)$ defined on $[0,1)$ such that as $t$ goes from 0 to 1 the point $a_{j}(t)$ traverses each curve in $a_{j}$ precisely once in the positive direction. Recall that $\alpha_{j}$ is a finite union of closed analytic Jordan curves. If $a_{j}$ contains $n$ contours, divide $[0,1)$ into $n$ half-open subintervals each of length $1 / n$. The points of subdivision introduced are $t_{k}=k / n(0 \leq k \leq n)$. Let $a_{j}$ restricted to the subinterval $\left[t_{k}, t_{k+1}\right)$ be an injective parametrization of a single contour of $\alpha_{j}$ which agrees with the orientation. Thus $a_{j}(t), t \in[0,1)$, is a path which traverses $\alpha_{j}$ once in the positive direction. Likewise, parametrize each part $\beta_{j}$ of $\gamma_{1}$ by $b_{j}(t), t \in[0,1) \quad(1 \leq j \leq m)$. 
These fixed parametrizations are used in continuing a harmonic conjugate of $u$ along a portion of $\partial \bar{R}$ in a consistent manner. We illustrate this for $\alpha_{0}$. Suppose $u^{*}$ is a local harmonic conjugate of $u$ defined near $a_{0}(0)$. Through harmonic continuation the function $u^{*}$ may be defined along the curve $a_{0}\left(\left[0, t_{1}\right)\right)$. The value $u^{*}\left(a_{0}\left(t_{1}^{-}\right)\right)$exists but need not be equal to $u^{*}\left(a_{0}(0)\right)$. Extend $u^{*}$ to the curve $a_{0}\left(\left[t_{1}, t_{2}\right)\right)$ as follows. First determine a conjugate function $u_{1}^{*}$ for $u$ in a neighborhood of $a_{0}\left(t_{1}\right)$ such that $u_{1}^{*}\left(a_{0}\left(t_{1}\right)\right)=u^{*}\left(a_{0}\left(t_{1}^{-}\right)\right)$and then continue $u_{1}^{*}$ harmonically along $a_{0}\left(\left[t_{1}, t_{2}\right)\right)$. In this manner we extend $u^{*}$ to all of $\alpha_{0}$ so that $u^{*}\left(a_{0}(t)\right)$ is a continuous function on $[0,1)$; in short, $u^{*}$ is continuous on $\alpha_{0}$. Functions are to be continued around $\alpha_{1}$ and the $\beta_{j}$ in a similar fashion. The fact that the integral of $* d u=d u^{*}$ over $\beta_{j}$ vanishes guarantees that $u^{*}$ returns to its initial value after traversing $\beta_{j}$ once. Explicitly, $u^{*}\left(b_{j}(t)\right)$ may be extended to the real line as a continuous function having period 1 .

The function $u^{*}\left(a_{0}(t)\right)$ strictly decreases from 0 to $-D(u)$ on $[0,1)$ provided $u^{*}$ is normalized so that $u^{*}\left(a_{0}(0)\right)=0$. Since $0 \leq u \leq 1$ on $\bar{R}$, in a relative neighborhood of each point of $\alpha_{0}$ the holomorphic function $f=u+i u^{*}$ has nonnegative real part and is purely imagininary on $a_{0}$. Therefore in terms of any local coordinate $z$ mapping such a neighborhood onto a relatively open subset of the upper half-plane, the function $f(z)$ has a purely imaginary derivative and $\operatorname{Im}\left\{f^{\prime}(z)\right\}<0$ on $a_{0}$. In other words, $d u^{*}=* d u<0$ along $\alpha_{0}$ so that $u^{*}$ is strictly decreasing. From

$$
D_{\bar{R}}(u)=\int_{\partial \bar{R}} u * d u=\int_{a_{1}} * d u=-\int_{\alpha_{0}} * d u,
$$

we see that $u^{*}$ decreases from 0 to $-D(u)$.

Two complementary subsets, $S$ and $C$, of the interval $(-D(u), 0]$ shall be defined. $C$ will be finite and with each $s \in S$ we shall associate a chain in $\mathcal{F}$. For each $s \in(-D(u), 0]$ a single level curve $l_{0}(s)$ of $u^{*}$ leaves $a_{0}$. Orient $l_{0}(s)$ so that $u$ increases in the positive direction. $l_{0}(s)$ is a curve along which $* d u=0$ and is an analytic Jordan arc unless it passes through a critical point of $u$. In case the connected level curve $l_{0}(s)$ passes through a critical point of $u$ in $\bar{R}$, place $s$ in $C$. If $l_{0}(s)$ does not cross any critical points of $u$, then it ends on either $\alpha_{1}$ or $\gamma_{1}$. When $l_{0}(s)$ terminates on $\alpha_{1}$, put $s$ in $S$ and write $c(s)$ in place of $l_{0}(s)$. Note that $c(s) \in \mathcal{F}$. Otherwise, $l_{0}(s)$ stops on some $\beta_{j} \subset \gamma_{1}$. Let $\zeta(s)$ denote the endpoint of $l_{0}(s)$ on $\beta_{j}$; our assumption about $l_{0}(s)$ guarantees that $\zeta(s)$ is not a critical point of $u$. Now move along $\beta_{j}$ in the positive direction following the path determined by $b_{j}$. The function $u^{*}$ is initially increasing as we continue $u^{*}$ from $\zeta(s)$ along $\beta_{j}$. Continue $u^{*}$ along $\beta_{j}$ until arriving at $\zeta^{\prime}(s)$, the first point for which $u^{*}\left(\zeta^{\prime}(s)\right)=u^{*}(\zeta(s))$. Such a point $\zeta^{\prime}(s)$ exists because the integral of $* d u$ over $\beta_{j}$ is 0 . For future reference we note that $\zeta^{\prime}(s)=$ $\zeta^{\prime}(t)$ if and only if $\zeta(s)=\zeta(t)$. If $\zeta^{\prime}(s)$ is a critical point of $u$, then put $s$ in $C$. If not, then the level curve $l_{1}(s)$ of $u^{*}$ which originates at $\zeta^{\prime}(s)$ moves into $R$ 
when oriented in the direction of increasing values of $u$. In fact, $* d u<0$ along $\beta_{j}$ near $\zeta^{\prime}(s)$ since $* d u>0$ along $\beta_{j}$ by $\zeta(s)$; this also shows that $\zeta^{\prime}(s) \neq$ $\zeta(s)$. Thus, $u+i u^{*}$ maps a relative neighborhood of $\zeta^{\prime}(s)$ onto a relative open set in $\left\{z: \operatorname{Re}\{z\} \geq c_{j}\right\}$ where $u \equiv c_{j}$ on $\beta_{j}$. The level curve $l_{1}(s)$ is called a continuation of $l_{0}(s)$. If $l_{1}(s)$ crosses a critical point of $u$, then $s$ goes in $C$. Otherwise, $u$ is strictly increasing on $l_{1}(s)$ so that the connected level curve $l_{1}(s)$ ends on either $\alpha_{1}$ or some $\beta_{k}$ with $k \neq j$. In the former case, write $c(s)=l_{0}(s)+l_{1}(s)$ and put $s$ in $S$. Again $c(s) \in \mathcal{F}$. In the latter we repeat the construction given above.

In this manner we see that each $s \in(-D(u), 0]$ is in either $C$ or $S$. For every $s \in S$ there is a finite chain $c(s)=l_{0}(s)+\cdots+l_{j(s)}(s)$ of connected level curves of $u^{*}$ obtained by successive continuation beginning with $l_{0}(s)$ and $c(s) \epsilon$ $\mathcal{F}$. For $s \neq t$ in $S$ the chains $c(s)$ and $c(t)$ are disjoint. For if $l_{j}(s) \cap l_{k}(t) \neq \emptyset$, then $l_{j}(s)=l_{k}(t)$ because they are level curves for the same harmonic function and do not pass through any critical points. If $j=k=0$, then $s=t$ is immediate.

Otherwise, $j, k \geq 1$ and $l_{j}(s), l_{k}(t)$ have the same initial point on some part of $\gamma_{1}$ so that $l_{j-1}(s)$ and $l_{k-1}(t)$ must have the same terminal point. But then $l_{j-1}(s)=$ $l_{k-1}(t)$. Continuing in this manner we find that $l_{0}(s)=l_{0}(t)$ and $s=t$. The set $C$ is finite because $u$ has only finitely many critical points on the compact surface $\bar{R}$ and through each such point pass only finitely many level curves of $u^{*}$.

Finally we establish the reverse inequality. Except at the finite number of critical points of $u$ the function $u+i u^{*}$ can be used as a local parameter and $\bar{R}$ can be paved with little rectangles determined by the level curves of $u$ and $u^{*}$. For any linear density $d s=\rho|d z|$ and $s \in S$,

$$
\int_{c(s)} \rho|d z|=\int_{c(s)} \rho d u \leq\left(\int_{c(s)} \rho^{2} d u\right)^{1 / 2} .
$$

Here we have used the facts that $* d u=0, d u>0$ along $c(s)$ and that the integral of $d u$ over $c(s)$ is 1 . Since $\{c(s): s \in S\} \subset \mathcal{F}$,

$$
L^{2}(\mathcal{F}, d s) \leq\left(\int_{c(s)} \rho d u\right)^{2} \leq \int_{c(s)} \rho^{2} d u .
$$

Integration over $S$ results in

$$
D_{\bar{R}}(u) L^{2}(\mathcal{F}, d s) \leq \int_{s \in S} \int_{C(s)} \rho^{2} d u d u^{*}=A(d s),
$$

so that $\lambda(\mathcal{F}) \leq D(u)^{-1}$, which completes the proof.

3. Extensions to noncompact bordered surfaces. Next we present three generalizations of Theorem 1 to the case in which $\bar{R}$ is a noncompact bordered Riemann surface having compact border $\partial \bar{R}$. In this section $\bar{R}$ is fixed and the contours of $\partial \bar{R}$ are partitioned into two nonempty disjoint sets, $\alpha_{0}$ and $\alpha_{1}$. 
(3.1) The first generalization corresponds to Theorem 1 when $\gamma_{1}=\emptyset$. In this situation we are interested in the extremal length of the family $\mathcal{F}$ of all curves in $\bar{R}$ which join $\alpha_{0}$ to $\alpha_{1}$; that is, the extremal distance of $\alpha_{0}$ and $\alpha_{1}$. The following result is known ([4, Theorem III. 3.1, p. 256], [8, Theorem 2A, p. 156]), but the proof is new and brief and is included for completeness.

Theorem 2. $\lambda(\mathfrak{F})=D_{\bar{R}}\left(u_{0}\right)^{-1}$ where $u_{0}$ is the barmonic function on $\bar{R}$ such that $u_{0}=0$ on $\alpha_{0}, u_{0}=1$ on $\alpha_{1}$ and $u_{0}=L_{0} u_{0}$ in a neighborbood of the ideal boundary of $\vec{R}$.

Proof. Let $\left\{\bar{R}_{n}\right\}_{n=1}^{\infty}$ be a regular exhaustion of $\bar{R}$; that is, $\left\{\bar{R}_{n}\right\}$ is a strictly increasing sequence of compact bordered Riemann surfaces such that $\bar{R}=\bigcup \bar{R}_{n}$ and $\partial \bar{R}_{n} \supset \partial \bar{R}=\alpha_{0} \cup \alpha_{1}$ for all $n$. Set $\gamma_{n}=\partial \bar{R}_{n} \sim \partial \bar{R}$, then $\left\{\alpha_{0}, \alpha_{1}, \gamma_{n}\right\}$ is a partition of $\partial \bar{R}_{n}$ into disjoint sets of contours with $\alpha_{0}, \alpha_{1}$ nonempty. Let $u_{n}$ be the harmonic function on $\bar{R}_{n}$ with the properties $u_{n}=0$ on $\alpha_{0}, u_{n}=1$ on $\alpha_{1}$ and $* d u_{n}=0$ along $\gamma_{n}$. If $\mathcal{F}_{n}$ is the family of all curves in $\bar{R}_{n} \sim \gamma_{n}$ joining $a_{0}$ to $a_{1}$, then Theorem 1 gives $\lambda\left(\mathcal{F}_{n}\right)=D\left(u_{n}\right)^{-1}$ where the Dirichlet integral is taken over $\bar{R}_{n}$. Now, $\mathcal{F}_{n} \subset \mathcal{F}_{n+1}$ for all $n$ and $\mathcal{F}=\mathcal{U F}_{n}$ so that $\lambda(\mathcal{F})=\lim \lambda\left(\mathcal{F}_{n}\right)$ [9, p. 321]. The sequence $\left\{u_{n}\right\}$ is uniformly convergent on compact subsets of $\bar{R}$ to $u_{0}$ and $D\left(u_{n}\right)$ converges to $D\left(u_{0}\right)\left[8\right.$, Theorem $2 \mathrm{~B}, \mathrm{p}$. 58]. Hence, $\lambda(\mathcal{F})=D\left(u_{0}\right)^{-1}$.

(3.2) The next extension of Theorem 1 is the analog of the case in which $\gamma_{0}=$ $\emptyset$ and $\gamma_{1}$ is given the identity partition; in other words, all contours of $\gamma_{1}$ belong to the same part. Let $\bar{R}^{\prime}$ be the Alexandroff one-point compactification of $\bar{R}$ and $\beta$ the point added to $\bar{R}$ to obtain $\bar{R}^{\prime}$. Given a sequence $\left\{\zeta_{n}\right\}$ of points in $\bar{R}$, write $\zeta_{n} \rightarrow \beta$ if for every compact set $E \subset \bar{R}$ there is an integer $N=N(E)$ with the property that $\zeta_{n} \notin E$ for all $n \geq N$. Similarly, if $l:[0,1) \rightarrow \bar{R}$ is a curve, then $l(t) \rightarrow$ $\beta$ as $t \rightarrow 1$ means that for each compact set $E \subset \bar{R}$ there is a $\delta>0$ such that $l(t) \notin E$ for $1-\delta<t<1$. Say that $l(t)$ clusters on $\beta$ as $t \rightarrow 1$ if there is a sequence $t_{n} \rightarrow 1$ with $l\left(t_{n}\right) \rightarrow \beta$. Let $\mathcal{F}^{\prime}$ be the family of curves in $\bar{R}^{\prime}$ connecting $\alpha_{0}$ to $\alpha_{1} . \lambda\left(\mathcal{F}^{\prime}\right)$ is the extremal length of the family of chains obtained by restricting each curve in $\mathcal{F}^{\prime}$ to $\bar{R}$. Since $\mathfrak{F}^{\prime} \supset \mathcal{F}$, it follows that $\lambda\left(\mathcal{F}^{\prime}\right) \leq \lambda(\mathfrak{F})$. Two related families $\mathcal{G}^{\prime}$ and $\mathcal{H}^{\prime}$ are needed. $\mathcal{G}^{\prime}$ is the family of chains $l$ such that either $l$ is a curve in $\bar{R}$ joining $\alpha_{0}$ to $\alpha_{1}$ or $l=l_{0}+l_{1}$ where $l_{j}:[0,1) \rightarrow \bar{R}$ is a curve with $l_{j}(0) \in \alpha_{j}$ and $l_{j}(t)$ clusters on $\beta$ as $t \rightarrow 1(j=0,1)$. $\mathcal{H}^{\prime}$ is the subfamily of $\bigcup^{\prime}$ consisting of the curves in $\bar{R}$ joining $\alpha_{0}$ to $\alpha_{1}$ together with the chains $l_{0}+l_{1}$ such that $l_{j}(t) \rightarrow \beta$ as $t \rightarrow 1$. If we define $l_{j}(1)=\beta$ in this case, then $l_{j}$ becomes a curve from $\alpha_{j}$ to $\beta$ in $\bar{R}^{\prime}$ and $l_{0}+l_{1}$ may be viewed as a curve in $\bar{R}^{\prime}$ joining $\alpha_{0}$ to $\alpha_{1}$. In this natural way we regard $\mathcal{H}^{\prime}$ as a subset of $\mathcal{F}^{\prime}$. Both $\mathcal{G}^{\prime}$ and $\mathcal{H}^{\prime}$ contain $\mathcal{F}$. The following result shows that $\mathcal{F}^{\prime}, \mathcal{G}^{\prime}$ and $\mathcal{H}^{\prime}$ are equivalent families for extremal length problems.

Proposition 1. $\lambda\left(\mathcal{F}^{\prime}\right)=\lambda\left(\varrho^{\prime}\right)=\lambda\left(\mathfrak{H}^{\prime}\right)$. 
Proof. The inclusion $\mathcal{H}^{\prime} \subset \mathcal{F}^{\prime}$ implies $\lambda\left(\mathcal{H}^{\prime}\right) \geq \lambda\left(\mathcal{F}^{\prime}\right)$. Every curve in $\mathcal{F}^{\prime}$ contains a chain in $\mathcal{H}^{\prime}$ so that $\lambda\left(\mathcal{F}^{\prime}\right) \geq \lambda\left(\mathcal{H}^{\prime}\right)$ [9, p. 321]. Therefore, $\lambda\left(\mathcal{F}^{\prime}\right)=\lambda\left(\mathcal{H}^{\prime}\right)$.

In order to establish $\lambda\left(\mathcal{H}^{\prime}\right)=\lambda\left(\mathscr{G}^{\prime}\right)$ it is sufficient to show that $\lambda\left(\mathscr{G}^{\prime} \sim \mathcal{H}^{\prime}\right)=\infty$ [8, Corollary 2A, p. 128]. The family $\mathcal{G}^{\prime} \sim \mathcal{H}^{\prime}$ has infinite extremal length because every chain in this family must oscillate. Let $\left\{\bar{R}_{n}\right\}$ be a regular exhaustion of $\bar{R}$ and set $\gamma_{n}=\partial \bar{R}_{n} \sim \partial \bar{R}$. About each contour of every $\gamma_{n}$ place an annulus which is contained in $\bar{R}_{n+1} \sim \bar{R}_{n-1}$ such that all of the annuli are pairwise disjoint. We shall prove that every chain in $\mathcal{G}^{\prime} \sim \mathcal{H}^{\prime}$ crosses some annulus infinitely often. Then $\lambda\left(\varrho^{\prime} \sim \mathcal{H}^{\prime}\right)=\infty$ follows because the family of chains each of which crosses at least one of the annuli infinitely often has infinite extremal length.

If $l \in \mathcal{Y}^{\prime} \sim \mathcal{H}^{\prime}$, then $l=l_{0}+l_{1}$ where both $l_{0}$ and $l_{1}$ cluster on $\beta$ but one of them does not actually tend to $\beta$ since $l \notin \mathcal{H}^{\prime}$. We consider the case in which $l_{0}$ does not approach $\beta$ as $t \rightarrow 1$. Then there is a sequence $\left\{s_{n}\right\}$ tending to 1 and an $\bar{R}_{N}$ such that $l_{0}\left(s_{n}\right) \in \bar{R}_{N}$ for all $n$. Since $l_{0}$ clusters on $\beta$, there is also a sequence $\left\{t_{n}\right\}$ for which $t_{n} \rightarrow 1$ and $l_{0}\left(t_{n}\right) \notin \bar{R}_{N+2}$ for all $n$. By extracting subsequences if necessary we may assume that $s_{n}<t_{n}<s_{n+1}$ for all $n$. In the interval $\left[s_{n}, t_{n}\right]$ the curve $l_{0}$ crosses at least one of the annular regions about the contours of $\gamma_{N+1}$. As there are but finitely many contours in $\gamma_{N+1}$, the curve $l_{0}$ must cross one of the annuli infinitely often.

This proposition simplifies the proof of next theorem.

Theorem 3. $\lambda\left(\mathcal{F}^{\prime}\right)=D_{\bar{R}}\left(u_{I}\right)^{-1}$ where $u_{i}$ is the barmonic function on $\bar{R}$ such that $u_{I}=0$ on $\alpha_{0}, u_{I}=1$ on $\alpha_{1}$ and $u_{I}=(I) L_{1} u_{I}$ in a neighborbood of the ideal boundary of $\bar{R}$.

Proof. The proof makes use of an extremal length technique ascribed to Beurling and developed by Wolontis [12, p. 591]. We employ the method in a manner related to the work of Strebel [10, pp. 10-11], Marden and Rodin [4, pp. 252-256] and Suita [11, pp. 239-242]. The proof is divided into two parts: first we show that $\lambda\left(\varrho_{n}^{\prime}\right) \rightarrow \lambda\left(\varrho^{\prime}\right)$ where $\mathscr{G}_{n}^{\prime}$ is a curve family associated with $\bar{R}_{n}$, part of a regular exhaustion of $\bar{R}$, and then we use this result to show that $\lambda\left(\varrho^{\prime}\right)=D\left(u_{I}\right)^{-1}$.

Let $\left\{\bar{R}_{n}\right\}$ be a regular exhaustion of $\bar{R}$ and set $\gamma_{n}=\partial \bar{R}_{n} \sim \partial \bar{R}$. Let $\oint_{n}^{\prime}$ be the collection of all chains $l$ such that either $l$ is a curve in $\bar{R}_{n} \sim \gamma_{n}$ joining $a_{0}$ to $\alpha_{1}$ or $l=l_{0}+l_{1}$ where $l_{j}$ is a curve in $\bar{R}_{n}$ connecting $\alpha_{j}$ to $\gamma_{n}(j=0,1)$. Because every chain in $\varrho_{n+1}^{\prime}$ contains a chain in $\mathscr{G}_{n}^{\prime}, \lambda\left(\mathscr{G}_{n}^{\prime}\right) \leq \lambda\left(\mathscr{G}_{n+1}^{\prime}\right)$ for all $n$ [9, p. 321], and hence $\lim \lambda\left(\mathcal{G}_{n}^{\prime}\right)$ exists. Also, every chain in $\mathcal{G}^{\prime}$ contains a chain in $\mathscr{G}_{n}^{\prime}$ so that $\lim \lambda\left(\mathscr{G}_{n}^{\prime}\right) \leq \lambda\left(\varrho^{\prime}\right)$. In order to prove that $\lim \lambda\left(\Theta_{n}^{\prime}\right)=\lambda\left(\varrho^{\prime}\right)$, it remains to show that $\lambda\left(\varrho^{\prime}\right) \leq \lim \lambda\left(\varrho_{n}^{\prime}\right)$. For this we may assume that $\lambda\left(\varrho^{\prime}\right)>0$ and $\lim \lambda\left(\mathscr{G}_{n}^{\prime}\right)<\infty$. Let $M>0$ be any number strictly less than $\lambda\left(\mathcal{G}^{\prime}\right)$, then it suffices to demonstrate that $M \leq \lim \lambda\left(\mathcal{G}_{n}^{\prime}\right)$. Now, there is a density $d s$ with $M<$ $L^{2}\left(\mathfrak{G}^{\prime}, d s\right) A(R, d s)^{-1}$. Since $\lambda\left(\mathfrak{G}^{\prime}\right)=\lambda\left(\mathcal{F}^{\prime}\right) \leq \lambda(\mathfrak{F})<\infty$, we may also assume that 
$A(\bar{R}, d s)>0$. Let $a_{n}=L\left(\mathscr{G}_{n}^{\prime}, d s\right)$, then $\left\{a_{n}\right\}$ is an increasing sequence. Moreover,

$$
a_{n}^{2} \leq A\left(\bar{R}_{n}, d s\right) \lambda\left(\varrho_{n}^{\prime}\right) \leq A(\bar{R}, d s) \lambda\left(\varrho_{n}^{\prime}\right) \leq A(\bar{R}, d s) \lim \lambda\left(\varrho_{n}^{\prime}\right),
$$

so that $a=\lim a_{n}$ is finite and $a^{2} \leq A(\bar{R}, d s) \lim \lambda\left(\varrho_{n}^{\prime}\right)$. Let $\epsilon>0$ be given. We shall prove there is an $l \in \mathcal{G}^{\prime}$ such that $L(l, d s)<a+5 \epsilon$. Then $L\left(\mathcal{G}^{\prime}, d s\right)<a+$

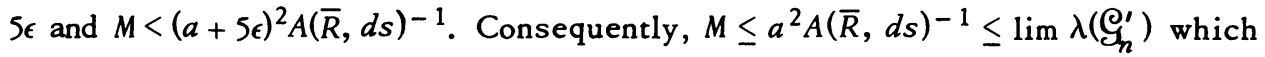
is the desired result.

Establishing the existence of $l$ is a bit involved. For every $n$ determine $l_{n} \epsilon$ $\oint_{n}^{\prime}$ with $L\left(l_{n}, d s\right)<a+\epsilon$. If for any value of $n$ the chain $l_{n}$ is actually a curve in $\bar{R}_{n} \sim \gamma_{n}$ joining $\alpha_{0}$ to $\alpha_{1}$, then we simply take $l=l_{n}$. Otherwise for every $n$, $l_{n}=l_{0 n}+l_{1 n}$ where $l_{j n}$ is a curve in $\bar{R}_{n}$ from $\alpha_{j}$ to $\gamma_{n}(j=0,1)$. Let $\zeta_{j n}(k)$ denote the first point of intersection of $l_{j n}$ with $\gamma_{k}$ for $k \leq n$. Initially we pass to a subsequence of $\left\{l_{j n}\right\}$ such that $\zeta_{j n}(1)$ converges to a point $\zeta_{j}(1) \in \gamma_{1}$ as $n \rightarrow$ $\infty$. This is possible because $\gamma_{1}$ is compact. We continue to denote this subsequence by $\left\{l_{j n}\right\}$. From this new sequence we again extract a subsequence, this time so that the points $\zeta_{j n}(2)$ converge to $\zeta_{j}(2) \in \gamma_{2}$. Continue in this manner. The diagonal sequence, which is still designated as $\left\{l_{j n}\right\}$, has the property that $\zeta_{j n}(k) \rightarrow_{n}$ $\zeta_{j}(k) \in \gamma_{k}$ for every $k$. Observe that in passing to a subsequence of $\left\{l_{j n}\right\}$ we have also gone to a subsequence of $\left\{\bar{R}_{n}\right\}$.

Let $V_{j}(k)$ be a parametric disk centered at $\zeta_{j}(k)$ such that the disks are all pairwise disjoint. Since the extremal length of the family of all Jordan curves in $V_{j}(k) \sim\left\{\zeta_{j}(k)\right\}$ which contain $\zeta_{j}(k)$ in their interior is $0[9, \mathrm{p} .325]$, there is a Jordan curve $c_{j}(k)$ in $V_{j}(k) \sim\left\{\zeta_{j}(k)\right\}$ enclosing $\zeta_{j}(k)$ with $L\left(c_{j}(k), d s\right)<\epsilon 2^{-k}$.

Let $\mathscr{D}_{j}(k)$ denote the family of all curves on $\bar{R}$ joining $c_{j}(k-1)$ to $c_{j}(k)$ for $k \geq 2$ and let $\mathscr{D}_{j}(1)$ be the collection of all curves on $\bar{R}$ connecting $\alpha_{j}$ to $c_{j}(1)(j=0,1)$. Then

$$
\sum_{k=1}^{\infty} L\left(\mathscr{X}_{j}(k), d s\right) \leq \lim _{n \rightarrow \infty} \inf L\left(l_{j n}, d s\right) .
$$

For each integer $k \geq 1$ let $\delta_{j}(k) \in \mathscr{D}_{j}(k)$ be such that $L\left(\delta_{j}(k), d s\right) \leq L\left(\mathscr{D}_{j}(k), d s\right)+$ $\epsilon 2^{-k}$. Let $\bar{c}_{j}(k)$ be a subarc of $c_{j}(k)$ which joins the end of $\delta_{j}(k)$ to the initial point of $\delta_{j}(k+1)$. Set

$$
l_{j}=\sum_{k=1}^{\infty}\left[\delta_{j}(k)+\bar{c}_{j}(k)\right] .
$$

If $l_{j}$ is parametrized in an appropriate manner, then $l=l_{0}+l_{1} \in \mathfrak{G}^{\prime}$. Also,

$$
\begin{aligned}
L\left(l_{j}, d s\right) & =\sum_{k=1}^{\infty}\left[L\left(\delta_{j}(k), d s\right)+L\left(\bar{c}_{j}(k), d s\right)\right] \\
& \leq 2 \epsilon+\underset{n \rightarrow \infty}{\liminf } L\left(l_{j n^{\prime}} d s\right) .
\end{aligned}
$$


From this inequality it follows that

$$
L(l, d s) \leq 4 \epsilon+\lim _{n \rightarrow \infty} \inf _{n}\left(l_{n}, d s\right)=a+5 \epsilon,
$$

so that $l \in \mathcal{G}^{\prime}$ is an appropriate choice.

So far we have shown that $\lim \lambda\left(\varrho_{n}^{\prime}\right)=\lambda\left(\varrho^{\prime}\right)$. Employing this result we may complete the proof. $\left\{\alpha_{0}, \alpha_{1}, \gamma_{n}\right\}$ is a partition of $\partial \bar{R}_{n}$ with $\alpha_{0}, \alpha_{1}$ nonempty. Let $\gamma_{n}$ be given the identity partition in which all contours belong to the same part. Take $\mathcal{F}_{n}$ to be the family of all finite chains in $\bar{R}_{n}$ which join $\alpha_{0}$ to $\alpha_{1}$, possibly via $\gamma_{n}$. Explicitly, $l \in \mathcal{F}$ if and only if either $l$ is a curve in $\bar{R} \sim \gamma_{n}$ from $\alpha_{0}$ to $\alpha_{1}$ or $l=\sum_{j=0}^{m} l_{j}$ where $m \geq 1, l_{j}$ is a curve connecting $\alpha_{j}$ to $\gamma_{n}(j=0,1)$ and $l_{j}$ is a curve joining any two contours in $\gamma_{n}(2 \leq j \leq m)$. By Theorem $1, \lambda\left(\mathcal{F}_{n}\right)=$ $D\left(u_{n}\right)^{-1}$ where $u_{n}$ is harmonic on $\bar{R}_{n}, u_{n}=0$ on $\alpha_{0}, u_{n}=1$ on $\alpha_{1}, u_{n}$ is a constant $c$ on $\gamma_{n}, 0 \leq c \leq 1$, and $\int_{\gamma_{n}} * d u=0$. Evidently, $\mathscr{G}_{n}^{\prime} \subset \mathcal{F}_{n}$ so that $\lambda\left(\mathscr{G}_{n}^{\prime}\right) \geq$ $\lambda\left(\mathcal{F}_{n}\right)$. But each chain in $\mathcal{F}_{n}$ contains a chain in $\mathfrak{G}_{n}^{\prime}$ so that $\lambda\left(\mathfrak{G}_{n}^{\prime}\right) \leq \lambda\left(\mathcal{F}_{n}\right)$. Therefore, $\lambda\left(\mathcal{G}_{n}^{\prime}\right)=\lambda\left(\mathfrak{F}_{n}\right)$. The sequence $\left\{u_{n}\right\}$ converges uniformly to $u_{I}$ on compact subsets of $\bar{R}$, and $D\left(u_{n}\right)$ tends to $D\left(u_{I}\right)$ [8, Theorem $2 A$, p. 58]. Hence, $\lambda\left(\mathcal{F}^{\prime}\right)=$ $\lambda\left(\Theta^{\prime}\right)=D\left(u_{I}\right)^{-1}$.

(3.3) The third generalization involves the Kerékjártó-Stoillow compactification $\bar{R}^{*}$ of $\bar{R}$; as is customary we view $\bar{R}$ as a subset of $\bar{R}^{*}$. Let $\mathcal{F} *$ be the family of all curves in $\bar{R}^{*}$ joining $a_{0}$ to $\alpha_{1} . \lambda(\mathfrak{F} *)$ is the extremal length of the family of chains obtained by restricting each curve in $\mathcal{F}^{*}$ to $\bar{R}$. Clearly, $\mathcal{F} \subset \mathcal{F}^{*} \subset \mathcal{F}^{\prime}$ which gives $\lambda\left(\mathfrak{F}^{\prime}\right) \leq \lambda\left(\mathfrak{F}^{*}\right) \leq \lambda(\mathfrak{F})$. This situation is analogous to Theorem 1 when $\gamma_{0}=\emptyset$ and $\gamma_{1}$ is given the canonical partition in which each contour in $\gamma_{1}$ constitutes a part of $\gamma_{1}$. The following result is a special case of a theorem of Marden and Rodin ([4, Theorem III. 3.1, p. 256], [8, Theorem 2A, p. 156]); it is given here for future reference. They obtain it from their version of Theorem 1.

Theorem 4. $\lambda(\mathfrak{F} *)=D_{\bar{R}}\left(u_{Q}\right)^{-1}$ where $u_{Q}$ is the barmonic function on $\bar{R}$ such that $u_{Q}=0$ on $\alpha_{0}, u_{Q}=1$ on $\alpha_{1}$ and $u_{Q}=(Q) L_{1} u_{Q}$ in a neighborbood of the ideal boundary of $\bar{R}$.

4. Reduced extremal distances. Let $R$ be a Riemann surface and $\zeta_{1}, \zeta_{2}$ two distinct points on $R$. We shall define three reduced extremal distances between $\zeta_{1}$ and $\zeta_{2}$ : one on $R$; another on $R^{\prime}$, the Alexandroff one-point compactification of $R$; and a third on $R^{*}$, the Kerékjártó-Stoilow compactification of $R$. The following notation will be standard in this section. Suppose that $z_{j}$ is a local coordinate defined in a neighborhood $N_{j}$ of $\zeta_{j}$ such that $z_{j}\left(\zeta_{j}\right)=0(j=1,2)$ and $N_{1} \cap N_{2}=$ $\emptyset$. For all sufficiently small positive values of $r$ the parametric disk $D_{j}(r)=$ $\left\{\zeta \in R:\left|z_{j}(\zeta)\right|<r\right\}$ and its boundary $\alpha_{j}(r)=\left\{\zeta \in R:\left|z_{j}(\zeta)\right|=r\right\}$ are both subsets of $N_{j}$

(4.1) Let $\mathcal{F}\left(r_{1}, r_{2}\right)$ designate the class of all curves on $R \sim\left[D_{1}\left(r_{1}\right) \cup D_{2}\left(r_{2}\right)\right]$ 
$=\bar{R}\left(r_{1}, r_{2}\right)$ which connect $\alpha_{1}\left(r_{1}\right)$ and $\alpha_{2}\left(r_{2}\right)$. The extremal length of the curve family $\mathcal{F}\left(r_{1}, r_{2}\right)$ is the extremal distance between the contours $\alpha_{1}\left(r_{1}\right)$ and $\alpha_{2}\left(r_{2}\right)$ on the bordered Riemann surface $\bar{R}\left(r_{1}, r_{2}\right)$.

Proposition 2. $\lambda\left(\mathcal{F}\left(r_{1}, r_{2}\right)\right)+(1 / 2 \pi) \log \left(r_{1} r_{2}\right)$ increases if either $r_{1}$ or $r_{2}$ decreases.

Proof. Suppose that $0<r_{j} \leq R_{j}$ and let $\mathbb{Q}\left(r_{j}, R_{j}\right)$ be the family of all curves in the annular region $D_{j}\left(R_{j}\right) \sim \mathrm{Cl}\left(D_{j}\left(r_{j}\right)\right)$ which join the inner boundary contour $\alpha_{j}\left(r_{j}\right)$ to the outer curve $\alpha_{j}\left(R_{j}\right)$. Every curve in $\mathcal{F}\left(r_{1}, r_{2}\right)$ contains a curve from each of the families $\mathfrak{Q}\left(r_{1}, R_{1}\right), \mathcal{F}\left(R_{1}, R_{2}\right)$ and $\mathfrak{Q}\left(r_{2}, R_{2}\right)$ and the supports of these three curve families are pairwise disjoint Borel sets. Hence [9, p. 321],

$$
\begin{aligned}
\left.\lambda \mathcal{F}\left(r_{1}, r_{2}\right)\right) & \geq \lambda\left(\mathbb{Q}\left(r_{1}, R_{1}\right)\right)+\lambda\left(\mathcal{F}\left(R_{1}, R_{2}\right)\right)+\lambda\left(\mathfrak{A}\left(r_{2}, R_{2}\right)\right) \\
& =\frac{1}{2 \pi} \log \frac{R_{1}}{r_{1}}+\lambda\left(\mathcal{F}\left(R_{1}, R_{2}\right)\right)+\frac{1}{2 \pi} \log \frac{R_{2}}{r_{2}},
\end{aligned}
$$

which establishes the proposition.

The preceding result guarantees that $\lambda\left(\mathcal{F}\left(r_{1}, r_{2}\right)\right)+(1 / 2 \pi) \log \left(r_{1} r_{2}\right)$ approaches a limit (possibly $+\infty)$ as $\left(r_{1}, r_{2}\right) \rightarrow(0,0)$; the limit is the supremum taken over $r_{1}, r_{2}$ positive and sufficiently small. The number

$$
\lambda\left(\zeta_{1}, \zeta_{2}\right)=\lim _{\left(r_{1}, r_{2}\right) \rightarrow(0,0)}\left[\lambda\left(\mathcal{F}\left(r_{1}, r_{2}\right)\right)+(1 / 2 \pi) \log \left(r_{1} r_{2}\right)\right]
$$

is called the reduced extremal distance between $\zeta_{1}$ and $\zeta_{2}$. For convenience set $\lambda\left(\zeta_{1}, \zeta_{2}\right)=0$ if $\zeta_{1}=\zeta_{2}$. The value of $\lambda\left(\zeta_{1}, \zeta_{2}\right)$ depends upon the choice of the local parameter $z_{j}$ at $\zeta_{j}$. This dependence will be made explicit later. This concept of reduced extremal distance between two points is related to, but distinct from, the reduced extremal distance between a point and a compact set as defined by Ahlfors and Beurling ([2, p. 244], [5, pp. 238-241]), as well as their definition of the perimeter of a compact set relative to a point [1, pp. 115-116].

(4.2) Let $p_{0}(\zeta)=p_{0}\left(\zeta_{;} \zeta_{1}, \zeta_{2}\right)$ be the $L_{0}$-principal function having a negative logarithmic singularity at $\zeta_{1}$ and a positive logarithmic singularity at $\zeta_{2}$. Thus, $p_{0}$ is harmonic on $R \sim\left\{\zeta_{1}, \zeta_{2}\right\}, p_{0}=L_{0} p_{0}$ in a neighborhood of the ideal boundary of $R$ and $p_{0}\left(z_{j}\right)+(-1)^{j} \log \left|z_{j}\right|$ has a removable singularity at $z_{j}=0$ $(j=1,2)$. Let $p_{0}\left\langle\zeta_{j}\right\rangle$ denote the value of the latter harmonic function at $z_{j}=0$; in other words,

$$
p_{0}\left\langle\zeta_{j}\right\rangle=\lim _{z_{j} \rightarrow 0}\left[p_{0}\left(z_{j}\right)+(-1)^{j} \log \left|z_{j}\right|\right] \quad(j=1,2) .
$$

The value of $p_{0}\left\langle\zeta_{j}\right\rangle$ is defined with respect to the fixed local coordinate $z_{j}$. In general, the value of $p_{0}\left\langle\zeta_{j}\right\rangle$ would be different if another local coordinate were used. 
Theorem 5. $\lambda\left(\zeta_{1}, \zeta_{2}\right)=(1 / 2 \pi)\left(p_{0}\left\langle\zeta_{2}\right\rangle-p_{0}\left\langle\zeta_{1}\right\rangle\right)$.

Proof. First we show that the right-hand side is an upper bound for $\lambda\left(\zeta_{1}, \zeta_{2}\right)$ by making use of a Riemann surface $\Omega(r)$ containing $\bar{R}(r, r)$ on which it is easier to compute the relevant extremal lengths. A similar type of argument is used in [5, Theorem 2.94, p. 243]. For small positive values of $r$ let

$$
m_{1}(r)=\min _{\zeta \in a_{1}(r)} p_{0}(\zeta), \quad m_{2}(r)=\max _{\zeta \in \alpha_{2}(r)} p_{0}(\zeta)
$$

When $r$ is sufficiently small, $m_{1}(r)<0<m_{2}(r)$ and $c_{j}(r)=\left\{\zeta: p_{0}(\zeta)=m_{j}(r)\right\}$ $(j=1,2)$ is an analytic Jordan curve. Orient $c_{j}(r)$ so that the component of the complement of $c_{j}(r)$ which contains $\zeta_{j}$ lies to the left; in other words, $c_{j}(r)$ is oriented positively with respect to the point $\zeta_{j}$. Notice that $c_{j}(r) \subset \mathrm{Cl}\left(D_{j}(r)\right)$. Set $\Omega(r)=\left\{\zeta: m_{1}(r) \leq p_{0}(\zeta) \leq m_{2}(r)\right\} . \Omega(r)$ is a bordered Riemann surface having compact border $c_{1}(r) \cup c_{2}(r)$ and $\Omega(r) \supset \bar{R}(r, r)$.

Let $\lambda\left(c_{1}(r), c_{2}(r)\right)$ denote the extremal distance of $c_{1}(r)$ and $c_{2}(r)$ on the surface $\Omega(r)$; that is, the extremal length of the family of all curves on $\Omega(r)$ which join $c_{1}(r)$ to $c_{2}(r)$. Because every such curve contains a subcurve belonging to $\mathcal{F}(r, r)$, it follows that $\lambda(\mathcal{F}(r, r)) \leq \lambda\left(c_{1}(r), c_{2}(r)\right)[9$, p. 321]. It is a simple matter to compute $\lambda\left(c_{1}(r), c_{2}(r)\right)$ in terms of the function $p_{0}$. The function $u(\zeta)=$ $\left[m_{2}(r)-p_{0}(\zeta)\right]\left[m_{2}(r)-m_{1}(r)\right]^{-1}$ is harmonic on $\Omega(r), 0$ on $c_{2}(r), 1$ on $c_{1}(r)$ and $u=L_{0} u$ in a neighborhood of the ideal boundary of $\Omega(r)$. By Theorem 2 the extremal length $\lambda\left(c_{1}(r), c_{2}(r)\right)$ is equal to the reciprocal of the Dirichlet integral $D(u)$ of $u$ taken over $\Omega(r)$. An application of Stokes' theorem results in

$$
\begin{aligned}
& {\left[m_{2}(r)-m_{1}(r)\right]^{2} D_{\boldsymbol{\Omega}(r)}(u)=D_{\Omega(r)}\left(p_{0}\right)} \\
& \quad=-\left[m_{1}(r) \int_{c_{1}(r)} * d p_{0}+m_{2}(r) \int_{c_{2}(r)} * d p_{0}\right]=-2 \pi\left[m_{1}(r)-m_{2}(r)\right] .
\end{aligned}
$$

In applying Stokes' theorem no term arises which corresponds to the ideal boundary since $p_{0}$ has $L_{0}$-behavior near the ideal boundary. (For future reference observe that $(I) L_{1}$ or $(Q) L_{1}$-behavior in a neighborhood of the ideal boundary yields the same conclusion.) Consequently, $\lambda\left(c_{1}(r), c_{2}(r)\right)=(1 / 2 \pi)\left\lfloor m_{2}(r)-m_{1}(r)\right]$. From

$$
\lim _{r \rightarrow 0}\left[m_{2}(r)-m_{1}(r)+\log \left(r^{2}\right)\right]=p_{0}\left\langle\zeta_{2}\right\rangle-p_{0}\left\langle\zeta_{1}\right\rangle
$$

it is clear that $\lambda\left(\zeta_{1}, \zeta_{2}\right) \leq(1 / 2 \pi)\left[p_{0}\left\langle\zeta_{2}\right\rangle-p_{0}\left\langle\zeta_{1}\right\rangle\right]$. The opposite inequality is obtained by a similar procedure but using an inner approximation to $\bar{R}(r, r)$ instead.

(4.3) Theorem 5 provides an easy means to determine the dependence of $\lambda\left(\zeta_{1}, \zeta_{2}\right)$ on the choice of local parameters at $\zeta_{1}$ and $\zeta_{2}$. Suppose that $w_{j}$ is a local coordinate defined in a neighborhood $M_{j}$ of $\zeta_{j}$ with $w_{j}\left(\zeta_{j}\right)=0$. Let the 
reduced extremal distance between $\zeta_{1}$ and $\zeta_{2}$ relative to these coordinates be denoted by $\lambda_{w}\left(\zeta_{1}, \zeta_{2}\right)$ and let $\lambda_{z}\left(\zeta_{1}, \zeta_{2}\right)$ designate the reduced extremal distance when computed with respect to the coordinates $z_{1}$ and $z_{2}$. Then we would obtain $\lambda_{w}\left(\zeta_{1}, \zeta_{2}\right)=(1 / 2 \pi)\left[q_{0}\left\langle\zeta_{2}\right\rangle-q_{0}\left\langle\zeta_{1}\right\rangle\right]$ where $q_{0}$ is harmonic on $R \sim\left\{\zeta_{1}, \zeta_{2}\right\}$; $q_{0}\left(w_{j}\right)+(-1)^{j} \log \left|w_{j}\right|$ is harmonic near $w_{j}=0$ and $q_{0}=L_{0} q_{0}$ in a neighborhood of the ideal boundary of $R$.

The functions $p_{0}$ and $q_{0}$ differ by a constant. Let $z_{j}\left(w_{j}\right)$ denote the conformal mapping of a neighborhood of $w_{j}=0$ onto a neighborhood of $z_{j}=0$ which is induced by the local coordinates $z_{j}$ and $w_{j}$. The function $p_{0}-q_{0}$ becomes harmonic on $R$ upon setting

$$
\begin{aligned}
& \left(p_{0}-q_{0}\right)\left(\zeta_{1}\right)=p_{0}\left\langle\zeta_{1}\right\rangle-q_{0}\left\langle\zeta_{1}\right\rangle+\log \left|z_{1}^{\prime}(0)\right|, \\
& \left(p_{0}-q_{0}\right)\left(\zeta_{2}\right)=p_{0}\left\langle\zeta_{2}\right\rangle-q_{0}\left\langle\zeta_{2}\right\rangle-\log \left|z_{2}^{\prime}(0)\right| .
\end{aligned}
$$

Since $p_{0}-q_{0}$ is now harmonic on $R$ and has $L_{0}$-behavior in a neighborhood of the ideal boundary of $R, p_{0}-q_{0}$ must be constant. In particular, its values at $\zeta_{1}$ and $\zeta_{2}$ coincide, which gives

$$
\lambda_{w}\left(\zeta_{1}, \zeta_{2}\right)=\lambda_{z}\left(\zeta_{1}, \zeta_{2}\right)-(1 / 2 \pi) \log \left|z_{1}^{\prime}(0) z_{2}^{\prime}(0)\right|
$$

This demonstrates that the definition of the reduced extremal distance $\lambda\left(\zeta_{1}, \zeta_{2}\right)$ is not a conformal invariant. It also shows that $\exp \left[-2 \pi \lambda\left(z_{1}, z_{2}\right)\right]\left|d z_{1}\right|\left|d z_{2}\right|$ is an invariant form.

(4.4) Other reduced extremal distances between two points can be introduced by using the compactifications $R^{\prime}$ and $R^{*}$ of $R$. Take $\mathcal{F}^{\prime}\left(r_{1}, r_{2}\right)$ (respectively $\left.\mathcal{F} *\left(r_{1}, r_{2}\right)\right)$ to be the collection of all curves in $R^{\prime} \sim\left[D_{1}\left(r_{1}\right) \cup D_{2}\left(r_{2}\right)\right]\left(R^{*} \sim\right.$ $\left.\left[D_{1}\left(r_{1}\right) \cup D_{2}\left(r_{2}\right)\right]\right)$ joining $\alpha_{1}\left(r_{1}\right)$ to $\alpha_{2}\left(r_{2}\right) . \lambda\left(\mathcal{F}^{\prime}\left(r_{1}, r_{2}\right)\right.$ ) (respectively $\lambda\left(F_{*} *\left(r_{1}, r_{2}\right)\right)$ ) is the extremal length of the family of chains obtained by restricting each curve in $\mathcal{F}^{\prime}\left(r_{1}, r_{2}\right)$ (respectively, $\left.\mathcal{F} *\left(r_{1}, r_{2}\right)\right)$ to $R$. The limits

$$
\begin{aligned}
& \lambda^{\prime}\left(\zeta_{1}, \zeta_{2}\right)=\lim _{\left(r_{1}, r_{2}\right) \rightarrow(0,0)}\left[\lambda\left(\mathcal{F}^{\prime}\left(r_{1}, r_{2}\right)\right)+(1 / 2 \pi) \log \left(r_{1} r_{2}\right)\right], \\
& \lambda^{*}\left(\zeta_{1}, \zeta_{2}\right)=\lim _{\left(r_{1}, r_{2}\right) \rightarrow(0,0)}\left[\lambda\left(\mathcal{F}^{*}\left(r_{1}, r_{2}\right)\right)+(1 / 2 \pi) \log \left(r_{1} r_{2}\right)\right]
\end{aligned}
$$

both exist and are always finite. The existence of the limits is established exactly as in Proposition 2. $\lambda^{\prime}\left(\zeta_{1}, \zeta_{2}\right)$ is the Alexandroff one-point compactification reduced extremal distance between $\zeta_{1}$ and $\zeta_{2} \cdot \lambda^{*}\left(\zeta_{1}, \zeta_{2}\right)$ is the reduced extremal distance between $\zeta_{1}$ and $\zeta_{2}$ relative to the Kerékjártó-Stoïlow compactification. Because $\mathscr{F}\left(r_{1}, r_{2}\right) \subset \mathcal{F}^{\prime}\left(r_{1}, r_{2}\right), \mathcal{F} *\left(r_{1}, r_{2}\right)$, it follows that $\lambda\left(\zeta_{1}, \zeta_{2}\right) \geq \lambda^{\prime}\left(\zeta_{1}, \zeta_{2}\right), \lambda^{*}\left(\zeta_{1}, \zeta_{2}\right)$. Also, if $R$ is compact, then $\mathcal{F}\left(r_{1}, r_{2}\right)=\mathcal{F}^{\prime}\left(r_{1}, r_{2}\right)=\mathcal{F} *\left(r_{1}, r_{2}\right)$ and $\lambda\left(\zeta_{1}, \zeta_{2}\right)=\lambda^{\prime}\left(\zeta_{1}, \zeta_{2}\right)=$ $\lambda^{*}\left(\zeta_{1}, \zeta_{2}\right)$ 
Both $\lambda^{\prime}\left(\zeta_{1}, \zeta_{2}\right)$ and $\lambda^{*}\left(\zeta_{1}, \zeta_{2}\right)$ are also related to principal functions. Let $p_{I}(\zeta)=p_{I}\left(\zeta ; \zeta_{1}, \zeta_{2}\right)$ and $p_{Q}(\zeta)=p_{Q}\left(\zeta ; \zeta_{1}, \zeta_{2}\right)$ be the $(I) L_{1}$ and $(Q) L_{1}$ principal functions, respectively, having the singularity $(-1)^{j+1} \log \left|z_{j}\right|$ at $\zeta_{j}$. The values $p_{I}\left\langle\zeta_{j}\right\rangle$ and $p_{Q}\left\langle\zeta_{j}\right\rangle$ are defined in the same manner as $p_{0}\left\langle\zeta_{j}\right\rangle$. A proof of the following theorem would just parallel the proof of Theorem 5 except that $(I) L_{1}$ or $(Q) L_{1}$ behavior must be substituted whenever $L_{0}$ behavior occurs in the original proof. In the course of proving Theorem 5 we noted that the proof still went through under these conditions.

Theorem 6.

$$
\begin{aligned}
& \lambda^{\prime}\left(\zeta_{1}, \zeta_{2}\right)=(1 / 2 \pi)\left[p_{1}\left\langle\zeta_{2}\right\rangle-p_{I}\left\langle\zeta_{1}\right\rangle\right], \\
& \lambda^{*}\left(\zeta_{1}, \zeta_{2}\right)=(1 / 2 \pi)\left[p_{Q}\left\langle\zeta_{2}\right\rangle-p_{Q}\left\langle\zeta_{1}\right\rangle\right] .
\end{aligned}
$$

5. Pseudometrics obtained from classes of harmonic functions. As usual $R$ is an arbitrary Riemann surface. $H D(R)$ is the class of all harmonic functions on $R$ which have finite Dirichlet integral $D(u) . H D_{1}(R)=\{u \in H D(R): D(u) \leq 1\} . K D(R)$ consists of those $u \in H D(R)$ which have the property that $\int_{y} * d u=0$ for all dividing cycles $\gamma$ on $R$. When $R$ is a region in the complex plane, every cycle is dividing and $K D(R)$ is the class of harmonic functions on $R$ which possess a finite Dirichlet integral and have a single-valued harmonic conjugate in $R$. Set $K D_{1}(R)=K D(R) \cap H D_{1}(R)$. Both $H D(R)$ and $K D(R)$ are vector spaces under pointwise addition and scalar multiplication. If any two functions in $H D(R)$ which differ by a constant are identified, then $H D(R)$ becomes a Hilbert space with norm $\|u\|=$ $D(u)^{1 / 2}$ and inner product $(u, v)=D(u, v)$, the mixed Dirichlet integral. The subspace $K D(R)$ is also a Hilbert space. The orthogonal complement of $K D(R)$ in $H D(R)$ is denoted by $H M(R)$ and is called the class of harmonic measures. Set $H M_{1}(R)=H M(R) \cap H D_{1}(R)$. (The orthogonal decomposition $H D(R)=H M(R) \oplus$ $K D(R)$ of the space $H D(R)$ is really just the orthogonal decomposition $\Gamma_{b e}=\Gamma_{b m}$ $\oplus \Gamma_{b e} \cap \Gamma_{b s e}^{*}$ in the space of exact harmonic differentials [8, p. 194].) The classes $H D(R), K D(R)$ and $H M(R)$ always contain the constant functions. In case one of these classes contain no additional functions, then we say $R$ is of class $O_{H D}, O_{K D}$ or $O_{H M}$, respectively.

There is a simple sufficient condition for a family of functions $\{u\}$ in $H D(R)$ to constitute a normal family. If $\{D(u)\}$ is uniformly bounded and for some point $\zeta_{0} \in R$ the set $\left\{u\left(\zeta_{0}\right)\right\}$ is also uniformly bounded, then $\{u\}$ is a normal family [8, p. 37].

(5.1) First we define a pseudometric associated with $H D(R)$. For $\zeta_{1}, \zeta_{2} \in R$ set

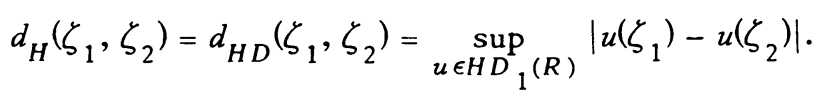


If $R \notin O_{H D}$, then it is sufficient to take the supremum over those $u \in H D(R)$ with $D(u)=1$. The number $d_{H}\left(\zeta_{1}, \zeta_{2}\right)$ is the best possible constant such that

$$
\left|u\left(\zeta_{1}\right)-u\left(\zeta_{2}\right)\right| \leq d_{H}\left(\zeta_{1}, \zeta_{2}\right) D(u)^{1 / 2}
$$

for all $u \in H D(R)$. The function $d_{H}$ is defined on $R \times R$ and assumes nonnegative real values. It is not immediate that $d_{H}$ is always finite. Notice that $d_{H}\left(\zeta_{1}, \zeta_{2}\right)$ $=\sup u\left(\zeta_{1}\right)$ where the supremum is taken over the compact normal family of all $u \epsilon$ $H D_{1}(R)$ with $u\left(\zeta_{2}\right)=0$. If $H D_{1}(R)$ is given the topology of uniform convergence on compact subsets of $R$, then the functional $u \rightarrow u\left(\zeta_{1}\right)$ is continuous on this compact subfamily. Hence, the least upper bound of the functional is actually attained and $d_{H}$ is finite-valued everywhere. The function $d_{H}$ has several interesting properties.

Proposition 3. (i) $d_{H}$ is a conformal invariant,

(ii) $d_{H}$ is a pseudometric on $R$,

(iii) $d_{H}$ is a monotone decreasing function of $R$,

(iv) the topology on $R$ generated by $d_{H}$ is coarser than the original topology,

(v) $d_{H}$ is a continuous function on $R \times R$,

(vi) $d_{H}$ is 2-subbarmonic on $R \times R$; in other words, $d_{H}$ is subbarmonic as a function of each variable separately.

Proof. (i) The conformal invariance of $d_{H}$ is a simple consequence of the fact that harmonicity and the Dirichlet integral are invariant under conformal mappings.

(ii) Clearly $\zeta_{1}=\zeta_{2}$ implies $d_{H}\left(\zeta_{1}, \zeta_{2}\right)=0$ and that $d_{H}$ is symmetric is obvious. The triangle inequality follows from the definition of $d_{H}$ and the ordinary triangle inequality.

(iii) Suppose that $S$ is a Riemann surface which contains $R$ and $\zeta_{1}, \zeta_{2} \in R$. Consider $H D_{1}(S)$ as a subset of $H D_{1}(R)$ by restricting to $R$ each function in $H D_{1}(S)$. Then $d_{H(S)}\left(\zeta_{1}, \zeta_{2}\right) \leq d_{H(R)}\left(\zeta_{1}, \zeta_{2}\right)$ is immediate from the definition of $d_{H}$.

(iv) Given $\zeta_{0} \in R$ and $\epsilon>0$ we must show that there is a neighborhood $N$ of $\zeta_{0}$ with the property that $N \subset\left\{\zeta_{:} d_{H}\left(\zeta_{0}, \zeta\right)<\epsilon\right\}$. Let $V$ be a parametric disk centered at $\zeta_{0}$ with local coordinate $z$. This means that $z\left(\zeta_{0}\right)=0$ and $z(V)=U$ is the unit disk in the complex plane. Employing the monotonicity and conformal invariance of $d_{H}$, we obtain

$$
d_{H(R)}\left(\zeta_{0}, \zeta_{1}\right) \leq d_{H(V)}\left(\zeta_{0}, \zeta_{1}\right)=d_{H(U)}\left(0, z_{1}\right)
$$

where $\zeta_{1} \in V$ and $z_{1}=z\left(\zeta_{1}\right) \in U$. It is possible to compute $d_{H(U)}\left(0, z_{1}\right)$ explicitly. Let $u \in H D(U)$, then there is a holomorphic function $f$ defined in $U$ the real part of which is $u$. If $f(z)=\sum_{n=0}^{\infty} a_{n} z^{n}$, then $D(u)=\pi \sum_{n=1}^{\infty} n\left|a_{n}\right|^{2}$. Using the Cauchy-Schwarz inequality, we have 


$$
\begin{aligned}
\left|u\left(z_{1}\right)-u(0)\right|^{2} & \leq\left|f\left(z_{1}\right)-f(0)\right|^{2}=\left|\sum_{n=1}^{\infty} a_{n} z_{1}^{n}\right|^{2} \\
& \leq \sum_{n=1}^{\infty} n\left|a_{n}\right|^{2} \sum_{n=1}^{\infty} \frac{\left|z_{1}\right|^{2}}{n}=\frac{1}{\pi} D(u) \log \left(1-\left|z_{1}\right|^{2}\right)^{-1}
\end{aligned}
$$

and equality holds if $u(z)=\log \left|1-\bar{z}_{1} z\right|^{-1}$. Therefore, $d_{H(U)}\left(0, z_{1}\right)=$ $\left[(1 / \pi) \log \left(1-\left|z_{1}\right|^{2}\right)^{-1}\right]^{1 / 2}$. Select $\delta>0$ so that $d_{H(U)}(0, z)<\epsilon$ for $|z|<\delta$. Then $N=\{\zeta:|z(\zeta)|<\delta\}$ is a suitable neighborhood of $\zeta_{0}$.

(v) Since $d_{H}\left(\zeta_{1}, \zeta_{2}\right)$ is defined as the supremum of the family of continuous functions $\left|u\left(\zeta_{1}\right)-u\left(\zeta_{2}\right)\right|$, where $u \in H D_{1}(R), d_{H}$ is certainly lower semicontinuous on $R \times R$. Establishing upper semicontinuity requires a normal family argument. Let $a$ be the upper limit of $d_{H}$ at the point $\left(\zeta_{1}, \zeta_{2}\right)$; we must show that $a \leq$ $d_{H}\left(\zeta_{1}, \zeta_{2}\right)$. Let the sequence $\left\{\zeta_{j n}\right\}$ be such that $\zeta_{j n} \rightarrow \zeta_{j}$ and $d_{H}\left(\zeta_{1 n}, \zeta_{2 n}\right) \rightarrow a$. There is a function $u_{n} \in H D_{1}(R)$ with $u_{n}\left(\zeta_{1 n}\right)=d_{H}\left(\zeta_{1 n}, \zeta_{2 n}\right)$ and $u\left(\zeta_{2 n}\right)=0$. Since $\zeta_{2 n} \rightarrow \zeta_{2}$ and the topology induced by $d_{H}$ is coarser than the original topology, we may assume that $d_{H}\left(\zeta_{2}, \zeta_{2 n}\right)<1$ for all $n$. Then $\left|u_{n}\left(\zeta_{2}\right)\right|=\mid u_{n}\left(\zeta_{2}\right)-$ $u_{n}\left(\zeta_{2 n}\right) \mid<1$ for every $n$ so that $\left\{u_{n}\right\}$ is a normal family. This permits us to suppose that $\left\{u_{n}\right\}$ is uniformly convergent on compact subsets of $R$ to a function $u \epsilon$ $H D_{1}(R)$. Then $u\left(\zeta_{2}\right)=\lim u_{n}\left(\zeta_{2 n}\right)=0$ and $a=\lim _{n \rightarrow \infty} u_{n}\left(\zeta_{1 n}\right)=u\left(\zeta_{1}\right) \leq d_{H}\left(\zeta_{1}, \zeta_{2}\right)$.

(vi) The supremum of a family of subharmonic functions is subharmonic provided the supremum is upper semicontinuous. For a fixed $\zeta_{2} \in R$ the function $\left|u(\zeta)-u\left(\zeta_{2}\right)\right|$ is subharmonic on $R$. The supremum $d_{H}\left(\zeta, \zeta_{2}\right)$ of all such functions in $H D_{1}(R)$ is continuous and hence subharmonic. By symmetry $d_{H}\left(\zeta_{1}, \zeta_{2}\right)$ is also subharmonic as a function of $\zeta_{2}$.

(5.3) In the same manner pseudometrics can be introduced on $R$ through the classes $K D(R)$ and $H M(R)$. Define

$$
\begin{aligned}
& d_{K}\left(\zeta_{1}, \zeta_{2}\right)=d_{K D}\left(\zeta_{1}, \zeta_{2}\right)=\sup _{u \in K D_{1}(R)}\left|u\left(\zeta_{1}\right)-u\left(\zeta_{2}\right)\right| \\
& d_{M}\left(\zeta_{1}, \zeta_{2}\right)=d_{H M}\left(\zeta_{1}, \zeta_{2}\right)=\sup _{u \in H M_{1}(R)}\left|u\left(\zeta_{1}\right)-u\left(\zeta_{2}\right)\right|
\end{aligned}
$$

Because $K D_{1}(R), H M_{1}(R) \subset H D_{1}(R)$, it is evident that $d_{K}\left(\zeta_{1}, \zeta_{2}\right), d_{M}\left(\zeta_{1}, \zeta_{2}\right) \leq$ $d_{H}\left(\zeta_{1}, \zeta_{2}\right)$. Both $d_{K}$ and $d_{M}$ share the properties listed for $d_{H}$ in Proposition 3.

(5.4) In general, $d_{H}, d_{K}$ and $d_{M}$ are just pseudometrics and not metrics. Clearly, $d_{H}\left(\zeta_{1}, \zeta_{2}\right)=0$ if and only if $u\left(\zeta_{1}\right)=u\left(\zeta_{2}\right)$ for all $u \in H D(R)$. Similar statements hold for $d_{K}$ and $d_{M}$. Both $d_{H}$ and $d_{K}$ are metrics when $R$ is a region in the complex plane having finite area $A(R)$. In this situation both harmonic functions $A(R)^{-1 / 2} x$ and $A(R)^{-1 / 2} y$ belong to $K D_{1}(R) \subset H D_{1}(R)$. When $z_{1}=x_{1}+i y_{1} \neq$ $z_{2}=x_{2}+i y_{2}$, it follows that

$$
d_{H}\left(z_{1}, z_{2}\right) \geq d_{K}\left(z_{1}, z_{2}\right) \geq A(R)^{-1 / 2} \max \left\{\left|x_{1}-x_{2}\right|,\left|y_{1}-y_{2}\right|\right\}>0 .
$$


For a general region $R$ in the extended complex plane $d_{K}$ is a metric if and only if $R \notin O_{K D}\left[1\right.$, Theorem 7, p. 113]. Thus, either $d_{K}$ is a metric or $d_{K} \equiv 0$ for subregions of the extended complex plane. Whether a similar result holds for $d_{H}$ is not known. Rodin [7] showed that for a nonplanar Riemann surface $d_{H}$ need not be a metric even though $R \notin O_{H D}$; the surface which he constructs has infinite genus. When $R$ is a simply connected Riemann surface $d_{M} \equiv 0$.

(5.5) The fact that $d_{H}$ is always finite shows that $u \rightarrow u\left(\zeta_{2}\right)-u\left(\zeta_{1}\right)$ is a bounded linear functional on the Hilbert space $H D(R)$. The Riesz representation theorem guarantees that there is a function $q_{H} \in H D(R)$, unique up to an additive constant, such that $u\left(\zeta_{2}\right)-u\left(\zeta_{1}\right)=D\left(u, q_{H}\right)$ for all $u \in H D(R)$. Clearly, $q_{H}$ depends upon the points $\zeta_{1}, \zeta_{2}$. Schwarz' inequality produces $\left|u\left(\zeta_{1}\right)-u\left(\zeta_{2}\right)\right|^{2} \leq$ $D(u) D\left(q_{H}\right)$ with equality if and only if $u=a q_{H}+b$ where $a$ and $b$ are constants. This proves that

$$
d_{H}\left(\zeta_{1}, \zeta_{2}\right)^{2}=D\left(q_{H}\right)=q_{H}\left(\zeta_{2}\right)-q_{H}\left(\zeta_{1}\right)
$$

It is known that $q_{H}=(1 / 2 \pi)\left(p_{0}-p_{I}\right)$ [9, Theorem $\left.2 \mathrm{H}, \mathrm{p} .39\right]$.

In the same manner, $d_{K}\left(\zeta_{1}, \zeta_{2}\right)^{2}=D\left(q_{K}\right)=q_{K}\left(\zeta_{2}\right)-q_{K}\left(\zeta_{1}\right)$ where $q_{K}=$ $(1 / 2 \pi)\left(p_{0}-p_{Q}\right) \in K D(R)$ [9, Theorem $2 \mathrm{H}$, p. 39]. Also, $d_{M}\left(\zeta_{1}, \zeta_{2}\right)^{2}=D\left(q_{M}\right)=$ $q_{M}\left(\zeta_{2}\right)-q_{M}\left(\zeta_{1}\right)$ where $q_{M}=(1 / 2 \pi)\left(p_{Q}-p_{I}\right) \in H M(R)$ [8, p. 103]. Notice that $q_{H}=$ $q_{K}+q_{M}$ is just the orthogonal decomposition of $q_{H} \in H D(R)$ relative to the orthogonal complements $K D(R)$ and $H M(R)$.

(5.6) Now we come to our main result which expresses the pseudometrics $d_{H}$, $d_{K}$ and $d_{M}$ in terms of the reduced extremal distances $\lambda, \lambda^{\prime}$ and $\lambda^{*}$.

Theorem 7.

$$
\begin{aligned}
& d_{H}\left(\zeta_{1}, \zeta_{2}\right)^{2}=\lambda\left(\zeta_{1}, \zeta_{2}\right)-\lambda^{\prime}\left(\zeta_{1}, \zeta_{2}\right), \\
& d_{K}\left(\zeta_{1}, \zeta_{2}\right)^{2}=\lambda\left(\zeta_{1}, \zeta_{2}\right)-\lambda^{*}\left(\zeta_{1}, \zeta_{2}\right), \\
& d_{M}\left(\zeta_{1}, \zeta_{2}\right)^{2}=\lambda^{*}\left(\zeta_{1}, \zeta_{2}\right)-\lambda^{\prime}\left(\zeta_{1}, \zeta_{2}\right),
\end{aligned}
$$

where $\lambda, \lambda^{\prime}$ and $\lambda^{*}$ are all computed with respect to the same local coordinates at $\zeta_{1}$ and $\zeta_{2}$.

Proof. Observe that $q_{H}\left(\zeta_{j}\right)=(1 / 2 \pi)\left(p_{0}\left\langle\zeta_{j}\right\rangle-p_{I}\left\langle\zeta_{j}\right\rangle\right)(j=1,2)$. Then by Theorems 5 and 6 and (5.5.1) we obtain $d_{H}\left(\zeta_{1}, \zeta_{2}\right)^{2}=\lambda\left(\zeta_{1}, \zeta_{2}\right)-\lambda^{\prime}\left(\zeta_{1}, \zeta_{2}\right)$. The results for $d_{K}$ and $d_{M}$ are established in the same manner.

Remark. Although the reduced extremal distances $\lambda, \lambda^{\prime}$ and $\lambda^{*}$ are not conformal invariants, the differences $\lambda-\lambda^{\prime}, \lambda-\lambda^{*}$ and $\lambda^{*}-\lambda^{\prime}$ are.

6. Applications. Using the preceding results we can obtain new extremal length tests to decide whether a Riemann surface is of class $O_{H D}$ or $O_{H M}$. These tests are analogs of a theorem of Rodin dealing with $O_{K D}[6]$, [8, Theorem $2 A$, p. 206]. From the definition of $d_{H}$ it is clear that $R \in O_{H D}$ if and only if $d_{H} \equiv 0$. 
By Theorem 7 a necessary and sufficient condition in order that $d_{H}$ vanish identically is for the two reduced extremal distances $\lambda\left(\zeta_{1}, \zeta_{2}\right)$ and $\lambda^{\prime}\left(\zeta_{1}, \zeta_{2}\right)$ to be equal for all $\zeta_{1}, \zeta_{2} \in R$. Of course, the reduced extremal distances must be computed relative to the same local coordinates at both $\zeta_{1}$ and $\zeta_{2}$. Similarly, $R \in O_{K D}$ (respectively, $\left.O_{H M}\right)$ if and only if $\lambda\left(\zeta_{1}, \zeta_{2}\right)=\lambda^{*}\left(\zeta_{1}, \zeta_{2}\right)$ (respectively, $\lambda^{\prime}\left(\zeta_{1}, \zeta_{2}\right)=$ $\left.\lambda^{*}\left(\zeta_{1}, \zeta_{2}\right)\right)$ for all $\zeta_{1}, \zeta_{2} \in R$. These simple observations form the bases for the various tests.

(6.1) Suppose that $E_{0}$ and $E_{1}$ are compact subsets of a Riemann surface $R$. Let $\mathcal{F}\left(E_{0}, E_{1}\right)$ be the family of all curves $c:[0,1] \rightarrow R$ such that $c(0,1) \subset R \sim$ $\left(E_{0} \cup E_{1}\right)$ and $c(j) \in E_{j}(j=0,1)$. Less precisely, $c$ is a curve in $R \sim\left(E_{0} \cup E_{1}\right)$ joining $E_{0}$ to $E_{1} . \lambda\left(\mathcal{F}\left(E_{0}, E_{1}\right)\right)$ is the extremal distance of $E_{0}, E_{1}$ and is denoted by $\lambda\left(E_{0}, E_{1}\right)$. The same extremal length would be obtained by considering all curves on $R$ with one endpoint in $E_{0}$ and the other in $E_{1}$. Recall that $R^{\prime}$ and $R^{*}$ denote the Alexandroff one-point and Kerékjártó-Stö̈low compactification of $R$, respectively. Let $\mathcal{F}^{\prime}\left(E_{0}, E_{1}\right)\left(\mathcal{F} *\left(E_{0}, E_{1}\right)\right)$ be the family of all curves in $R^{\prime} \sim\left(E_{0} \cup E_{1}\right)\left(R^{*} \sim\right.$ $\left.\left(E_{0} \cup E_{1}\right)\right)$ joining $E_{0}$ to $E_{1} . \lambda^{\prime}\left(E_{0}, E_{1}\right)\left(\lambda^{*}\left(E_{0}, E_{1}\right)\right)$ is the extremal length of the family of chains obtained by restricting each curve in $\mathcal{F}^{\prime}\left(E_{0}, E_{1}\right)\left(\mathcal{F}^{*}\left(E_{0}, E_{1}\right)\right)$ to $R$.

(6.2) Rodin established the following result [6].

Theorem 8. A necessary and sufficient condition that $\lambda\left(E_{0}, E_{1}\right)=\lambda^{*}\left(E_{0}, E_{1}\right)$ for all pairs of compact sets $E_{0}, E_{1}$ of $R$ is that $R \in O_{K D}$.

This theorem has analogs for both $O_{H D}$ and $O_{H M}$.

Theorem 9. A necessary and sufficient condition that $\lambda\left(E_{0}, E_{1}\right)=\lambda^{\prime}\left(E_{0}, E_{1}\right)$ (respectively, $\lambda^{\prime}\left(E_{0}, E_{1}\right)=\lambda^{*}\left(E_{0}, E_{1}\right)$ ) for all pairs of compact sets $E_{0}, E_{1}$ of $R$ is that $R \in O_{H D}$ (respectively, $O_{H M}$ ).

Proof. The proof of sufficiency is an adaptation of Rodin's, while the necessity proof is new and also yields a simpler proof in the case of his result. Thus, these three tests for $O_{H D}, O_{K D}$ and $O_{H M}$ may be derived in a uniform manner. We shall establish the result in detail only for the $O_{H D}$ test since the case dealing with $O_{H M}$ just parallels the given proof.

To prove the necessity, assume that the extremal distances $\lambda\left(E_{0}, E_{1}\right), \lambda^{\prime}\left(E_{0}, E_{1}\right)$ are always equal. Take distinct points $\zeta_{1}, \zeta_{2} \in R$; it suffices to show that $\lambda\left(\zeta_{1}, \zeta_{2}\right)=$ $\lambda^{\prime}\left(\zeta_{1}, \zeta_{2}\right)$. Let $z_{j}$ be a local parameter defined in a neighborhood of $\zeta_{j}$ with $z_{j}\left(\zeta_{j}\right)=0$. For $r$ positive and small, $E_{0}=\mathrm{Cl}\left(D_{1}(r)\right)$ and $E_{1}=\mathrm{Cl}\left(D_{2}(r)\right)$ are disjoint compact subsets of $R$. Note that $\mathcal{F}\left(E_{0}, E_{1}\right)=\mathfrak{F}(r, r)$ and $\mathscr{F}^{\prime}\left(E_{0}, E_{1}\right)=\mathcal{F}^{\prime}(r, r)$ in this case. Consequently, $\lambda(\mathcal{F}(r, r))=\lambda\left(\mathcal{F}^{\prime}(r, r)\right)$ so that $\lambda\left(\zeta_{1}, \zeta_{2}\right)=\lambda^{\prime}\left(\zeta_{1}, \zeta_{2}\right)$.

Similarly, $\lambda^{\prime}\left(E_{0}, E_{1}\right)=\lambda^{*}\left(E_{0}, E_{1}\right)$ implies that $\lambda^{\prime}\left(\zeta_{1}, \zeta_{2}\right)=\lambda^{*}\left(\zeta_{1}, \zeta_{2}\right)$ and $R$ $\epsilon O_{H M}$. Also, if $\lambda\left(E_{0}, E_{1}\right)=\lambda^{*}\left(E_{0}, E_{1}\right)$ for all compact $E_{0}, E_{1} \subset R$, then $\lambda\left(\zeta_{1}, \zeta_{2}\right)$ $=\lambda^{*}\left(\zeta_{1}, \zeta_{2}\right)$ and $R \in O_{K D}$. This is the short new proof of half of Rodin's result. 
The sufficiency is more complicated. Suppose that $R \in O_{H D}$ and let $E_{0}, E_{1}$ be compact subsets of $R$. If $E_{0} \cap E_{1} \neq \emptyset$, then $\lambda\left(E_{02} E_{1}\right)=\lambda^{\prime}\left(E_{0}, E_{1}\right)=\infty$. Therefore we may assume that $E_{0}$ and $E_{1}$ are disjoint. First we deal with the simple situation in which $R \sim\left(\left(E_{0} \cup E_{1}\right)^{0}\right)=\bar{W}$ is a bordered Riemann surface having compact border $\partial E_{0} \cup \partial E_{1}$. Then by Theorems 2 and $3, \lambda\left(E_{0}, E_{1}\right)=D_{W}\left(u_{0}\right)^{-1}$ and $\lambda^{\prime}\left(E_{0}, E_{1}\right)=D_{W}\left(u_{I}\right)^{-1}$ where $u_{0}, u_{I}$ are harmonic on $\bar{W}, u_{0}=u_{I}=0$ on $\partial E_{0}, u_{0}=$ $u_{I}=1$ on $\partial E_{1}$, and $u_{0}=L_{0} u_{0}, u_{I}=(I) L_{1} u_{I}$ in a neighborhood of the ideal boundary of $\bar{W}$ (which is also a neighborhood of the ideal boundary of $R$ ). But $(I) L_{1}=L_{0}$ because $R \in O_{H D}\left[8\right.$, Theorem $1 A$, p. 201]. Therefore, $u_{0}=u_{I}$ and $\lambda\left(E_{0}, E_{1}\right)=$ $\lambda^{\prime}\left(E_{0}, E_{1}\right)$.

If $\lambda^{*}\left(E_{0}, E_{1}\right)$ were being considered in this situation, then, from Theorem 4, $\lambda^{*}\left(E_{0}, E_{1}\right)=D_{W}\left(u_{Q}\right)^{-1}$ where $u_{Q}$ is harmonic on $\bar{W}, u_{Q}=0$ on $\partial E_{0}, u_{Q}=1$ on $\partial E_{1}$, and $u_{Q}=(Q) L_{1} u_{Q}$ in a neighborhood of the ideal boundary of $\bar{W}$. Rodin's result here uses the fact that $R \in O_{K D}$ if and only if $L_{0}=(Q) L_{1}[8$, Theorem $1 \mathrm{~B}$, p. 201]. The case dealing with $O_{H M}$ uses the result that $R \in O_{H M}$ if and only if $(I) L_{1}=(Q) L_{1}[8$, Theorem 1C, p. 202].

Next, we establish the result when $R \sim\left(E_{0} \cup E_{1}\right)$ is connected. In this situation $R \sim\left(E_{0} \cup E_{1}\right)$ is a Riemann surface and may be expressed as an increasing union of compact bordered Riemann surfaces. Let $\left\{\bar{R}_{n}\right\}$ be an increasing sequence of bordered Riemann surfaces having compact border $\partial \bar{R}_{n}$ such that (i) $R \sim$ $\left(E_{0} \cup E_{1}\right) \supset \bar{R}_{n}$ for all $n$, (ii) $R=\bigcup \bar{R}_{n}$ and (iii) each component of $R \sim \bar{R}_{n}$ meets one of $E_{0}$ and $E_{1}$ but not both. Divide the contours of $\partial \bar{R}_{n}$ into two disjoint sets $\alpha_{0 n}$ and $\alpha_{1 n}$ according as the contours bound components of $R \sim \bar{R}_{n}$ which meet $E_{0}$ or $E_{1}$. The first portion of the proof guarantees that $\lambda\left(\alpha_{0 n}, \alpha_{1 n}\right)=\lambda^{\prime}\left(\alpha_{0 n}, \alpha_{1 n}\right)$. Now, by using the extremal length technique originated by Beurling, it can be shown that $\lambda\left(\alpha_{0 n}, \alpha_{1 n}\right) \rightarrow \lambda\left(E_{0}, E_{1}\right)$ and $\lambda^{\prime}\left(\alpha_{0 n}, \alpha_{1 n}\right) \rightarrow \lambda^{\prime}\left(E_{0}, E_{1}\right)$. Hence, $\lambda\left(E_{0}, E_{1}\right)=$ $\lambda^{\prime}\left(E_{0}, E_{1}\right)$ in this situation.

Finally, the general case is handled by applying this last result to each component of $R \sim\left(E_{0} \cup E_{1}\right)$ and then using the fact that the reciprocal of extremal length is additive over families of curves having disjoint supports [5, Theorem 2.9, p. 78].

Corollary. $H D(R)=K D(R)$ if and only if $\lambda^{\prime}\left(E_{0}, E_{1}\right)=\lambda^{*}\left(E_{0}, E_{1}\right)$ for all pairs of compact sets $E_{0}, E_{1}$ in $R$.

Proof. $H D(R)=K D(R)$ if and only if $R \in O_{H M}$ [8, Theorem 1C, p. 202].

Given a compact set $E \subset R$ let $\lambda(E, \beta)$ denote the extremal length of the family $\mathcal{F}^{\prime}(E)$ of curves $c:[0,1) \rightarrow R$ such that $c(0) \in E$ and $c(t) \rightarrow \beta$ as $t \rightarrow 1$ where $\beta$ is the point added to $R$ to form $R^{\prime}$.

Corollary. If $\lambda(E, \beta)=\infty$ for every compact set $E \subset R$, then $R \in O_{H D}$. 
Proof. Let $E_{0}, E_{1}$ be compact subsets of $R$. It suffices to show that $\lambda(\mathcal{F})=$ $\infty$ where $\mathcal{F}=\mathcal{F}^{\prime}\left(E_{0}, E_{1}\right) \sim \mathcal{F}\left(E_{0}, E_{1}\right)$ since then $\lambda\left(E_{0}, E_{1}\right)=\lambda^{\prime}\left(E_{0}, E_{1}\right)$. Because every curve in $\mathcal{F}$ contains a subcurve in $\mathcal{F}^{\prime}\left(E_{j}\right)(j=0,1)$, it follows that $\lambda(\mathfrak{F}) \geq$ $\lambda\left(E_{j}, \beta\right)=\infty$.

Let $O_{G}$ be the class of Riemann surfaces which do not possess a Green's function. $R \in O_{G}$ if and only if $\lambda^{\prime}(E, \beta)=\infty$ for all compact sets $E \subset R$ [8, Remark 1, p. 200]. For a planar Riemann surface $R \in O_{G}$ if and only if $R \in O_{H D}$ [8, Theorem IF, p. 203]. In general, $O_{G} \varsubsetneqq O_{H D}$ [3, pp. 256-264].

7. Examples. Explicit computations may be given in the case of a hyperbolic simply connected Riemann surface.

(7.1) First consider the unit disk $U$. Then $H D(U)=K D(U), p_{I}=p_{Q}$ and $\lambda^{\prime}=$ $\lambda^{*}$. Given $z_{1}, z_{2} \in U$,

$$
\begin{aligned}
& p_{I}\left(z ; z_{1}, z_{2}\right)=g\left(z, z_{2}\right)-g\left(z, z_{1}\right)=\log \left|\frac{\left(z-z_{1}\right)\left(1-\bar{z}_{2} z\right)}{\left(z-z_{2}\right)\left(1-\bar{z}_{1} z\right)}\right|, \\
& p_{0}\left(z ; z_{1}, z_{2}\right)=N\left(z, z_{2}\right)-N\left(z, z_{1}\right)=\log \left|\frac{\left(z-z_{1}\right)\left(1-\bar{z}_{1} z\right)}{\left(\bar{z}-z_{2}\right)\left(1-\bar{z}_{2} z\right)}\right|,
\end{aligned}
$$

where $g\left(z, z_{j}\right)$ and $N\left(z, z_{j}\right)$ are Green's and Neumann's function, respectively, for $U$ having logarithmic singularity at $z_{j}$. Hence,

$$
q_{H}\left(z ; z_{1}, z_{2}\right)=\frac{1}{\pi} \log \left|\frac{1-\bar{z}_{1} z}{1-\bar{z}_{2} z}\right| .
$$

If $z-z_{j}$ is used as a local coordinate at $z_{j}$, then we obtain

$$
\begin{aligned}
\lambda\left(z_{1}, z_{2}\right) & =\frac{1}{2 \pi} \log \frac{\left|z_{1}-z_{2}\right|^{2}\left|1-\bar{z}_{1} z_{2}\right|^{2}}{\left(1-\left|z_{1}\right|^{2}\right)\left(1-\left|z_{2}\right|^{2}\right)} \\
\lambda^{\prime}\left(z_{1}, z_{2}\right) & =\frac{1}{2 \pi} \log \frac{\left|z_{1}-z_{2}\right|^{2}\left(1-\left|z_{1}\right|^{2}\right)\left(1-\left|z_{2}\right|^{2}\right)}{\left|1-\bar{z}_{1} z_{2}\right|^{2}} \\
d_{H}\left(z_{1}, z_{2}\right)^{2} & =\frac{1}{\pi} \log \frac{\left|1-\bar{z}_{1} z_{2}\right|^{2}}{\left(1-\left|z_{1}\right|^{2}\right)\left(1-\left|z_{2}\right|^{2}\right)} .
\end{aligned}
$$

These results are easily transformed to an arbitrary hyperbolic simply connected region in the complex plane. Let $z=f(w)$ map $R$ conformally onto $U$. Then $d_{H(R)}\left(w_{1}, w_{2}\right)=d_{H(U)}\left(f\left(w_{1}\right), f\left(w_{2}\right)\right)$ and, if $w-w_{j}$ is used as a local coordinate at $w_{j}$, then 


$$
\lambda_{R}\left(w_{1}, w_{2}\right)=\lambda_{U}\left(f\left(w_{1}\right), f\left(w_{2}\right)\right)-(1 / 2 \pi) \log \left|f^{\prime \prime}\left(w_{1}\right) f^{\prime}\left(w_{2}\right)\right|
$$

with a similar result holding for $\lambda_{R}^{\prime}\left(z_{1}, z_{2}\right)$. If $R$ is a Riemann surface and $z=$ $f(w)$ maps $R$ conformally onto $U$, then $\lambda_{R}\left(w_{1}, w_{2}\right)=\lambda_{U}\left(f\left(w_{1}\right), f\left(w_{2}\right)\right)$ if $f(w)$ $f\left(w_{j}\right)$ is used as a local coordinate at $w_{j}$.

(7.2) In a hyperbolic simply connected region $R, d_{H}$ is a conformally invariant metric and may be compared with the Poincaré hyperbolic metric $d$. The following results are readily established for $U$ :

$$
d_{H}\left(z_{1}, z_{2}\right)^{2}=(2 / \pi) \log \cosh d\left(z_{1}, z_{2}\right) \text {, }
$$

$$
d\left(z_{1}, z_{2}\right) \geq \pi^{1 / 2} d_{H}\left(z_{1}, z_{2}\right) \text { and equality holds if and only if } z_{1}=z_{2}
$$

$$
\lim _{z \rightarrow z_{0}} \frac{\pi^{1 / 2} d_{H}\left(z, z_{0}\right)}{\left|z-z_{0}\right|}=\frac{1}{1-\left|z_{0}\right|^{2}}=\lim _{z \rightarrow z_{0}} \frac{d\left(z, z_{0}\right)}{\left|z-z_{0}\right|}
$$

If $R$ is an arbitrary Riemann surface and $R \notin O_{H D}$, then $U$ is the universal covering surface for $R$ so that the hyperbolic metric $d$ may be introduced on $R$. The simple relationship (i) between $d$ and $d_{H}$ no longer holds on $R$. For instance, let $R=\{z: 0<|z|<1\}$, then $H D(R)=H D(U)$ because $z=0$ is a removable singularity for every function in $H D(R)$. Therefore, $d_{H(U)}=d_{H(R)}$, but for any fixed $z_{0} \in R$, the hyperbolic distance $d\left(z, z_{0}\right) \rightarrow \infty$ as $z \rightarrow 0$ ( $d$ is the hyperbolic metric on $R$ not in $U$ ).

\section{REFERENCES}

1. L. V. Ahlfors and A. Beurling, Conformal invariants and function-theoretic null-sets, Acta Math. 83 (1950), 101-129. MR 12, 1971.

2. - Conformal invariants. Construction and applications of conformal maps,

Proc. Sympos. Nat. Bur. Standards Appl. Math. Ser., no. 18, U. S. Government Printing Office, Washington, D. C., 1952. MR 14, 861.

3. L. V. Ahlfors and L. Sario, Riemann surfaces, Princeton Math. Series, no. 26, Princeton Univ. Press, Princeton, N. J., 1960. MR 22 \#5729.

4. A. Marden and B. Rodin, Extremal and conjugate extremal distance on open Riemann surfaces with applications to circular-radial slit mappings, Acta Math. 115 (1966), 237-269. MR 34 \#2862.

5. M. Ohtsuka, Dirichlet problem, extremal length and prime ends, Van Nostrand Reinhold Math. Studies, no. 22, Van Nostrand Reinhold, New York, 1970.

6. B. Rodin, Extremal length and removable boundaries of Riemann surfaces, Bull. Amer. Math. Soc. 72 (1966), 274-276. MR 32 \#265.

7. - On the span of a Riemann surface, Bull. Amer. Math. Soc. 76 (1970), 340341. MR $40 \# 5853$.

8. B. Rodin and L. Sario, Principal functions, Van Nostrand, Princeton, N. J., 1968. MR 37 \#5378.

9. L. Sario and K. Oikawa, Capacity functions, Die Grundlehren der math. Wissenschaften, Band 149, Springer-Verlag, New York, 1969. MR 40 \#7441.

10. K. Strebel, Die extremale Distanz zweier Enden einer Riemannschen Fläche, Ann. Acad. Sci. Fenn. Ser. A. I. No. 179 (1955), 21 pp. MR 16, 917.

11. N. Suita, On continuity of extremal distance and its applications to conformal mappings, Ködai Math. Sem. Rep. 21 (1969), 236-251. MR 41 \#3734. 
12. V. Wolontis, Properties of conformal invariants, Amer. J. Math. 74 (1952), 587-606. MR 14, 36.

DEPARTMENT OF MATHE MATICS, UNIVERSITY OF CALIFORNIA, SAN DIEGO, LA JOLLA, CALIFORNIA 92037

DEPARTMENT OF MATHEMATICS, UNIVERSITY OF MINNESOTA, MINNEAPOLIS, MINNESOTA 55455

Current address: Department of Mathematics, University of Cincinnati, Cincinnati, Ohio 45221 